Review

\title{
Entropy Production: Its Role in Non-Equilibrium Thermodynamics
}

\author{
Rosa Maria Velasco, Leopoldo Scherer García-Colín and Francisco Javier Uribe * \\ Departamento de Física, Universidad Autónoma Metropolitana-Iztapalapa, Apartado Postal 55-534, \\ C.P. 09340, México, Distrito Federal, Mexico \\ * Author to whom correspondence should be addressed; E-Mail: paco@xanum.uam.mx; \\ Tel.: +52-55-580-44949; Fax: +52-55-580-44611.
}

Received: 7 December 2010; in revised form: 31 December 2010 / Accepted: 4 January 2011 /

Published: 7 January 2011

\begin{abstract}
It is unquestionable that the concept of entropy has played an essential role both in the physical and biological sciences. However, the entropy production, crucial to the second law, has also other features not clearly conceived. We all know that the main difficulty is concerned with its quantification in non-equilibrium processes and consequently its value for some specific cases is limited. In this work we will review the ideas behind the entropy production concept and we will give some insights about its relevance.
\end{abstract}

Keywords: Boltzmann equation; entropy production; fluctuations; uncompensated heat

\section{Historical Remarks}

The concept of entropy, indispensable for dealing with the subject of entropy production, is certainly one of the most abused, and misunderstood concepts in theoretical physics. Since it is also unquestionably related to the concept of irreversibility, we believe that, even for the $\mathrm{n}^{\mathrm{t} h}$ time, it is convenient to briefly summarize in this review both its origins and what we believe is its correct position in this field, as well as its pertinent conceptualization.

So, let us go back to June 12, 1824 when Sadi Carnot published his treatise on heat engines [1]. His main contribution was that for an engine drawing heat from a hot reservoir the amount of work that may be obtained from the engine could never reach a $100 \%$ efficiency. There would always be a heat lost to a cold reservoir which he knew would arise from friction, noise and vibration. Nevertheless he was able to predict that the efficiency of a "Carnot engine" would depend only on the temperature difference 
within the engine. It was left to William Thomson, later Lord Kelvin, to prove in 1852 that this was indeed the case and that a thermodynamic or universal temperature $(T)$ could be defined and shown to be numerically equal to the empirical temperature $(\theta)$. It is also worth mentioning that it was around this time that Lord Kelvin himself already spoke of the natural tendency of the existence of energy dissipation in natural processes [2]. This fact was already well known to Newton [3].

The last part of this work was due to R. Clausius who, between 1854 and 1875 made three important contributions [4]. The first one, well known to any student of thermostatics, is the fact that in a reversible process the heat transferred $\pm d Q_{r}$ between the system and a heat reservoir whose temperature is $\theta$, the integral over the whole cycle of $\frac{d Q_{r}}{\theta}$ vanishes. Moreover he proved that $\theta=T$ and that $\frac{d Q_{r}}{T}$ is the differential of a state function $S$ which he later called the "entropy". Hence $S$ is defined only for systems known to be in their equilibrium states. Secondly he defined two "transformations", the conversion of heat $Q$ into work $W$ and its inverse, and the transfer of heat $\Delta Q$ from a hot to a cold body and the opposite. He then showed that in a reversible cycle [5],

$$
\mathcal{N} \equiv-\int \frac{d Q_{r}}{T}=0
$$

with the convention $d Q_{r}<0$ if given by the system and $d Q_{r}>0$ if absorbed. He then proceeded to state that for irreversible processes, which he never carefully defined, the "compensation" $\mathcal{N}$ is such that the heat transferred from a hot to a cold body and the conversion of work into heat required no compensation whereas the inverse processes did. Thirdly, his great step was to show that for transformations which occur in any cyclic process $\mathcal{N}$ must be non-negative or,

$$
\mathcal{N} \equiv-\int \frac{d Q}{T} \geq 0
$$

so that if the cycle has a reversible path connecting two arbitrary equilibrium states 1 and 2 and an irreversible one connecting the same two states, then trivially

$$
\mathcal{N}+\int_{(1)}^{(2)} \frac{d Q}{T}=S(2)-S(1)
$$

which is, for closed systems the most general statement of the second law thermodynamics. Clearly, for an infinitesimal arbitrary process, Equaqion (3) reads

$$
d S+d \mathcal{N}=\frac{d Q}{T}
$$

and for obvious dimensional requirements, $d \mathcal{N}$ may be written as

$$
d \mathcal{N}=\frac{d Q^{\prime}}{T}
$$

where $d Q^{\prime}$ is now called "uncompensated heat". This heat as already pointed out by Carnot, Kelvin and Clausius himself, arises from the dissipative effects naturally present in any real process (friction!) and it is rather unfortunate that the appropriate term for such effect was not kept but substituted by its present one "entropy production", which, if carefully analyzed, means nothing. 
The last contribution to this effect came from a very distinguished student of Clausius, Max Planck, who clearly defined thermodynamical irreversibility and proved a wonderful theorem [6]: "If any process that has been identified as irreversible is shown to be reversible, then all irreversible processes must be reversible." Noticing next that in nature there exist no process free from friction he gives a full significance to Clausius' compensation attribute leaving a very important open question, namely, what is the nature and how can we quantify $\mathcal{N}$ ? Attempts to answer this question are deeply associated with the concept of irreversibility itself and to the contents of which is now known as irreversible thermodynamics. This term is obviously repetitive since the second one ought to be enough. But uses and habits prevail so we shall keep the full term in what follows.

\section{Irreversible Thermodynamics}

It appears that the first explicit reference to the existence of irreversible processes in macroscopic physics is due to J. B. Fourier. In the stage of developing what later became his magnificent book "La Théorie Analytique du Chaleur" [7] he established clearly that the process of transferring heat from a hot to a cold body is irreversible and further, that the amount of heat transferred per unit area and unit of time is proportional to the temperature gradient existing between the bodies. He then proceeded to study heat transfer in simple geometries, spheres and cylinders and showed that for steady states,

$$
\nabla \cdot(\kappa \nabla T)=0
$$

$\kappa$ being a property of the material, and for non-steady states,

$$
\frac{\partial T}{\partial t}=D_{T} \nabla^{2} T
$$

if the thermal diffusivity $D_{T}=\kappa / \rho c_{V}$ where $\rho$ is the density and $c_{V}$ is the specific heat at constant volume, is independent of the position.

In opposition to microscopic dynamics where classically Newton's equations are invariant under time reflections, $t \rightarrow-t$, Equation (7) is not, a signature characterizing an irreversible (dynamical) process.

In $1854 \mathrm{G}$. Kirchhoff reached similar results for the electrical charge flow. If the electric field is $\mathbf{E}=-\nabla \phi$ where $\phi$ is the electric potential, then the steady state of a conductor is characterized by the equation

$$
\nabla \cdot(\xi \nabla \phi)=0
$$

where $\xi$ is the electrical conductivity, implying that there is no charge accumulation on it. It is rather striking that Equations (6) and (8) are nothing but the Euler-Lagrange equations for the variational principle

$$
\delta \int \xi(\mathbf{r})(\nabla \phi)^{2} d V=0
$$

subject to the condition that $\delta \phi(\mathbf{r})=0$ on the boundaries of $V$, and the same for the temperature. One therefore wonders why the study of irreversible processes did not follow the route of its relatives, namely, mechanics and electrodynamics for which the equations of motion can be obtained from Lagrangians or Hamiltonians through variational principles. In fact, this path was followed by Gibbs in thermostatics [8] but has rarely been pursued in thermodynamics. In short, is it possible to formulate 
variational principles for what we may call a non-equilibrium entropy and obtain information about how fast and along which trajectory an irreversible process occurs? Very few and restricted attempts have been made along this line by Onsager [9,10], Gyarmati [11], and Ziegler [12] but until today a convincing solid reply to this question remains unknown. This claim is somewhat unfair. Almost forty years ago the mathematical physicist M. A. Biot [13] published a delightful monograph dealing precisely with the formulation of variational principles to deal with the problem of heat transfer, both in linear and non-linear systems. As he showed in the appendix of this work the method is applicable to other fields including irreversible thermodynamics. The main question is how to construct variational principles for field variables such that the resulting Lagrangian equations are equivalent to a minimum dissipation principle. This is achieved through a function which, in many cases turns out to be proportional to the standard entropy production term. This result brings to two facts: first, the absolutely unphysical association of the true cause of irreversibility, namely Clausius' uncompensated heat, with the entropy production which has obscured the heart of the problem; second, the rather frequent assertion stating that very little work has been done in this direction. Our opinion is that Biot's result and plausible generalizations have simply being ignored.

A clear example of the above method is the relationship between the Bénard instability and entropy production, whereby one obtains a thermodynamic interpretation of dissipative structures. Although this kind of analysis and other similar examples are so far restricted to linear regimes [14], the extensions to non-linear cases could be approached using Biot's ideas.

In order to keep the language as precise as possible we shall use the symbol $S$ for the equilibrium value of the entropy, or its local equilibrium counterpart as it will soon show up. The thermodynamic function which will play the role of an entropy, provided it can be defined with precision, will be denoted by $\eta$ per unit mass so that $\rho \eta$ will act as an a non-equilibrium entropy per unit volume and $\int \rho \eta d V$ where the integration is over the whole volume of the system, is the sought "non-equilibrium" entropy $S^{N E}$. We warn the reader that the physical content of all this terminology remains still to be carefully clarified.

We may now return to examine the origins of modern non-equilibrium thermodynamics which bring us back to the differential form of Equation (3) which in turn, together with Equation (3) may be written as

$$
T d S^{N E}-đ Q=đ Q^{\prime}>0
$$

for an arbitrary process. Clearly, Clausius entropy $S$ cannot appear in (10) since the process is not necessarily a reversible one. Nevertheless, in 1923 the Belgian physical chemist T. de Donder took Equation (10) and wrote it as $[15,16]$

$$
T \frac{d S}{d t}-\frac{d Q}{d t}=\frac{d Q^{\prime}}{d t} \geq 0
$$

which is, in general, not valid unless we appeal to the local equilibrium assumption, as we shall discuss later. Using the definition of affinity, Gibbs equation for chemical reactions and the first law of thermodynamics also written as a time dependent equation, he was able to show that

$$
\frac{d Q^{\prime}}{d t} \equiv \sigma=\frac{A}{T} J_{c}
$$

where $J_{c}$, the so called "chemical flux" is proportional to the time rate of change of the "degree of advancement" of a chemical reaction $[15,16]$. The term $\frac{A}{T}$ that he identified with the chemical 
force inducing the reaction and the time rate of change of Clausius' uncompensated heat became the "entropy production", a rather unfortunate term since it hides the origin of $₫ Q^{\prime}$, namely, dissipation. Yet this "flux-force" relation became the fundamental relation that for over forty years dominated the development of irreversible thermodynamics, and in many ways spread the significance of the term "entropy production".

In this connection it is interesting to mention a specific case for which Clausius uncompensated heat can be readily calculated. Indeed consider a homogeneous reactive system undergoing an isochoric-isothermal chemical reaction involving $r$ components and assume that the local equilibrium assumption (LEA) holds true. Then for the change in the entropy one may write

$$
d S=d_{i} S+d_{e} S
$$

where $d_{e} S^{N E}=\frac{d Q}{T}$, assuming the existence of a universal temperature $T$. The quantity $d_{i} S^{N E}$ is related to the internal dissipation processes occurring during such process and thus is somehow related to Clausius' uncompensated heat, and $S$ is the local equilibrium entropy. Gibbs equation reads

$$
d S=\frac{1}{T} d H-\frac{1}{T} \sum_{r} \mu_{r} d \eta_{r}
$$

$\mu_{r}$ being the chemical potential for the $r^{\underline{t h}}$ component with $\eta_{r}$ moles.

Now $\frac{1}{T} d H=\frac{1}{T}(d Q)_{p}=d_{e} S$, is the change in the entropy due to the heat transferred between the system and its surroundings. Thus

$$
d S=d_{e} S-\frac{1}{T} \sum_{r} \mu_{r} d \eta_{r}
$$

By definition, Gibbs free energy changes in time according to

$$
\frac{d G}{d t}=\frac{d H}{d t}-T \frac{d S}{d t}
$$

so that combining Equations (15) and (16) and using the definition of chemical affinity,

$$
A=\sum_{r} \mu_{r} d \eta_{r}
$$

we get that

$$
\frac{d G}{d t}=\frac{d A_{T, V}}{d t}=\frac{d_{i} S}{d t}
$$

But according to Garfinkle $[17,18]$ for such reactions,

$$
\frac{d A_{T, V}}{d t}=A_{r e f}\left(\frac{1}{t}-\frac{1}{t_{K}}\right)
$$

where $A_{\text {ref }}$ is a reference value for the affinity and $t_{K}$ the time (finite) in which the reaction reaches equilibrium. From Equations (18) and (19) we see that the total amount of heat produced by the "internal" friction in the reaction is

$$
\Delta_{i} S=A_{\text {ref }}\left[\ln \left(t_{0} / t_{K}\right)-\left(\frac{t_{0}}{t_{K}}-1\right)\right]
$$


where $t_{0}$ is an initial time clearly different from zero since collisions must take place before the reaction starts. Clausius uncompensated heat would be $T \Delta_{i} S$, in principle measurable in the laboratory.

The theories that were set forward using this idea where pioneered by Onsager in $1931[19,20]$ and afterwards modified and adapted to a great variety of systems by Eckart in 1940 [21,22], Meixner during 1942-1943 [23], Prigogine in 1947 [24], de Groot (1952) [25], and finally summarized in the well known monograph of de Groot and Mazur in 1962 [26]. Since all this work is well known to the thermodynamics audience and further, it has been also analyzed within the framework of more recent developments in several review articles [27-29]. We will limit ourselves here to outline the most relevant ideas underlying such work, emphasizing those which are relevant to the purposes of this paper.

The starting point of these theories now referred to as Linear Irreversible Thermodynamics (LIT) is to write for the change in the non-equilibrium entropy $S^{N E}$ for any process occurring in the system, either open or closed, that

$$
d S^{N E}=d_{e} S^{N E}+d_{i} S^{N E}
$$

For the whole volume $V$ occupied by the system,

$$
S^{N E} \equiv \int \rho \eta d V
$$

and

$$
\frac{d S^{N E}}{d t}=-\int_{A} \mathbf{J}_{\eta, t} \cdot d \mathbf{a}
$$

where $\mathbf{J}_{\eta, t}$ is the total entropy flowing through the boundaries $A$ of the system. Moreover,

$$
\frac{d_{i} S^{N E}}{d t}=-\int \sigma d V
$$

where $\sigma$ (multiplied by $T$ ) is Clausius' uncompensated heat per unit volume. Pointwise, Equations (22-24) lead to the well known "entropy balance" equation, namely,

$$
\frac{\partial(\rho \eta)}{\partial t}+\nabla \cdot \mathbf{J}_{\eta, t}=\sigma
$$

which is indeed an "empty" equation since none of the quantities present has been given a clear physical significance. In LIT this is achieved by assuming that the "local equilibrium assumption" holds true [30]. In simple words this assumption establishes that the validity of the usual thermostatic relationships among state (equilibrium) variables hold true at every space point $\mathbf{r}$ of the system and any time $t$. Thus, $S^{N E} \rightarrow S$, Clausius' entropy, $\eta \equiv \mathrm{s}(\mathbf{r}, t)$,

$$
\mathbf{J}_{\eta, t} \equiv \frac{\mathbf{J}_{q}}{T}+\rho \mathbf{s} \mathbf{u}
$$

where $\mathbf{J}_{q}$ is the conventional heat flux, $\mathbf{u}$ is the hydrodynamical velocity, $\rho \equiv \rho(\mathbf{r}, t)$ and $\sigma$ turns out to be, as mentioned before, a bilinear form in the fluxes $\left(\mathbf{J}_{i}\right)$ and their corresponding forces $\left(X_{i}\right)$. Whence

$$
\sigma=\sum_{i=1}^{r} \mathbf{J}_{i}: X_{i}
$$


In Equation (27) the symbol : denotes the appropriate tensorial contraction. Finally one assumes that the theory is valid for small values of the "gradients", a restriction contained in the well known linear relationship between forces and fluxes which transforms Equation (27) into a quadratic form

$$
\sigma=\sum_{i, j=1}^{r} L_{i j} X_{i} X_{j}
$$

where $L_{i j}$ is the matrix of the transport coefficients. $\sigma$ is referred to as the "entropy production" in the system and as Clausius conjectured, it arises from the dissipative effects which are irremediably present in any irreversible process. Besides, $L_{i j}=L_{j i}$, as it was proved by Onsager himself, a fact well known as Onsager's reciprocity theorem [31]. Further, in LIT one either assumes or proves, using in general experimental information about the transport coefficients, that $\sigma>0$, which is consistent with the second law of thermodynamics. The vast number of processes to which this formalism may be applied is so well documented that it is completely unnecessary to extend ourselves in this direction. One additional comment is however pertinent. The term "entropy production" associated to $\sigma$ is unfortunate. In fact it is meaningless as to speak of the production of energy, pressure, volume or any other thermostatic variable. Being what it is, the concept of dissipation should have remained attached to it, $\int T(\mathbf{r}, t) \sigma(\mathbf{r}, t) d \mathbf{r}$ is precisely, the total amount of "uncompensated heat" arising from friction and/or the dissipative mechanisms. It is precisely that amount of heat that we can measure in the laboratory, entropy, even in its local meaning is not a measurable quantity.

At this stage one reaches the confrontation of how to go beyond LIT whose limitations are also well known and also very nicely summarized in recent review articles on the subject $[27,28]$. The first, most fundamental question is precisely the physical meaning of the so-called "non-equilibrium entropy". If this is not achieved then what we may understand about irreversible process remains in the dark. In 1969, using rather attractive ideas about the operation of electrical networks, Meixner conjectured [32] that for processes occurring in non-equilibrium systems $S^{N E}$ either cannot be uniquely defined or if it can, it may be done in an infinite number of ways. Curiously enough, using similar arguments it has been shown that for a linear planar electric network, the currents that appear in the network, governed by Kirchhoff's law, are distributed in such a way that the maximum entropy production state is achieved [36]. In this calculation the local equilibrium assumption enters in a rather disguised way since $d_{i} S$ in Equation (21) is identified with the equilibrium entropy and $\frac{d \sigma}{d t}$ with $\frac{1}{T} \frac{d Q}{d t}$ although in this last expression, energy dissipation appears in its usual form, Joule's heat $R I^{2}$.

From the microscopic view and for closed systems the second possibility was shown already over twenty years ago to be the most probable one [33,34]. Thus it appears that Shannon's information entropy would be the most suitable candidate for this purpose but in that case $\sigma$ would have to be carefully interpreted.

Another alternative that has prevailed in the last thirty years consists of several approaches all known under the generic name of continuous or rational thermodynamics, although continuum and rational may not be exactly synonymous. They can be in general classified as entropy theories and entropy free thermodynamics. The latter seem to have serious deficiencies so we shall not worry about them 
here $[27,28]$. The former ones basically start from the assumption that the so-called Gibbs-Duhem equation holds true in a finite volume of the system, namely

$$
\frac{d}{d t} \int_{V} \rho \eta d V+\int_{A} \mathbf{\Phi} \cdot d \mathbf{a}-\int_{V} \rho f_{\eta} d V=\int \rho \sigma d V>0
$$

where $\rho \eta$ is defined in Equation (22), $\boldsymbol{\Phi} \equiv \mathbf{J}_{\eta, t}$ the total entropy flux and $f_{\eta}$ is the entropy source per unit mass. Thus a non-equilibrium entropy is assumed to exist and further the total uncompensated heat (here $\sigma$ is per unit mass) is assumed to obey the second law of thermodynamics. In some of these theories Equation (29) is assumed to hold also locally so that

$$
\frac{\partial}{\partial t}(\rho \eta)+\nabla \cdot \Phi-\rho f_{\eta} \equiv \rho \sigma \geq 0
$$

The two most successful members of these theories are those of Coleman and Noll and of I. Müller. Both have been thoroughly discussed, evaluated and compared in excellent recent reviews to which the interested reader is referred for all pertinent details [27,28]. Nonetheless one has to accept that neither one completely fulfills the requirements of a unique, solid thermodynamic theory of irreversibility. Many loopholes and fine points are still at large and more work will be required in this direction. In the following two sections we will provide, first a more microscopic basis specifically for Clausius' uncompensated heat and second, some examples of a variety of phenomena illustrating the relevance of this concept.

Before proceeding with the following sections, one last note of caution must be given. This note concerns with essentially three terms that frequently appear in the literature, namely, the theorem of minimum entropy production as stated in LIT [24,26], the maximum entropy production principle, and the maximum entropy formalism. When read on a first glance they appear to be, if not contradictory, somewhat confusing. Let us briefly recall their origins. The minimum entropy production theorem was first proved by Prigogine and in essence establishes that if in a system a subset of the irreversible forces acting on it are kept constant, the fluxes which are generated by the remaining set of available forces are zero and the entropy production in the system is a minimum. Proofs, examples and long discussions about the limitations and applications of this result are available in the literature [24,26]. Further, that the theorem is a simple corollary of the Onsager-Gyarmati's variational method. A particular case of the maximum entropy production principle (MEPP) set up originally by these authors may be found in Reference [37]. Further, the MEPP method and the maximum entropy formalism (MEP) are practically synonymous. In the words of one of its stronger adepts, E. T. Jaynes [38], the method is not as ambitious as a physical theory which asks for explanations of how systems behave. Here we simply ask a more modest question: "Given the practical information that we have from observations, what are the best predictions we can make of observable phenomena?" Thus, concerning the subject of this paper, one is not seeking an explanation of irreversibility but limits the answer to describe and predict observable facts. These ideas are very old [39]. Their probabilistic roots date back to Bayes and Laplace and were brought into physics by Maxwell, Boltzmann, Gibbs, and Shannon. The concept of entropy production appears only when MEF itself is used to deal with irreversible processes and in this context has been extensively used and described for a large variety of cases. We shall come back to it in the forthcoming sections in a very careful way. 


\section{Entropy Production and Kinetic Theory}

As we pointed out, the concept of entropy is, if not the most, certainly one of the most abused, misunderstood and polemic concepts in theoretical physics. In the kinetic theory of dilute gases the situation is somewhat less critical since the so-called $H$ theorem provides an explicit form for the so-called $H$ functional that in equilibrium is related to the Sackur-Tetrode formula for the equilibrium entropy of a classical perfect gas. However, the question of irreversibility (which is not restricted to dilute gases) closely tied to the $H$ theorem has provided extensive discussions in the literature that even in these days are present, making us to believe that the discussions will continue in the future. In part this situation can be explained because we have new discoveries and tools that were unavailable to previous researchers; the emergence of chaos in deterministic systems and the wide use of computational techniques[40], for example.

Let us start by making some brief historical remarks that, again, are useful to put the ideas in perspective [41]. According to Chapman and Cowling [42], in 1858 Clausius introduced the concept of mean free path, although earlier studies started with Daniel Bernoulli in 1738. Later on, in 1859, Maxwell obtained formulae for the transport coefficients using the concept of mean free path, introduced the concept of velocity distribution function $(f)$, and obtained the form of the equilibrium distribution function $f^{(0)}$.

Coming back to the origins of entropy production in the kinetic theory of dilute gases, in 1872 Boltzmann with his $H$-theorem improved Maxwell's proof for the form of $f^{(0)}$, the celebrated Maxwell-Boltzmann distribution function, by using an integro-differential equation, now named the Boltzmann equation;

$$
\frac{\partial f}{\partial t}+\mathbf{c} \cdot \nabla_{\mathbf{r}} f+\mathbf{F} \cdot \nabla_{\mathbf{c}} f=J(f, f)
$$

where $J(f, f)$ represents the collision term whose explicit form is given below, $\mathbf{c}$ is the molecular velocity, and $\mathbf{F}$ is the external force per unit mass (acceleration). The collision term is given by,

$$
J(f, f)=\int d \mathbf{e}^{\prime} \int d \mathbf{c}_{1} g \Sigma(g, \chi)\left(f\left(\mathbf{r}, \mathbf{c}^{\prime}, t\right) f\left(\mathbf{r}, \mathbf{c}_{1}^{\prime}, t\right)-f(\mathbf{r}, \mathbf{c}, t) f\left(\mathbf{r}, \mathbf{c}_{1}, t\right)\right)
$$

This term gives the rate of change of the distribution function due to binary collisions. It consists of a gain term corresponding to molecules with initial velocities $\mathbf{c}^{\prime}$ and $\mathbf{c}_{1}^{\prime}$ that after colliding have final velocities $\mathbf{c}$ and $\mathbf{c}_{1}$, and a lost term in which molecules with initial velocities $\mathbf{c}$ and $\mathbf{c}_{1}$ collide and end up with final velocities $\mathbf{c}^{\prime}$ and $\mathbf{c}_{1}^{\prime}$. Since one is interested in calculating all possible situations in which one of the molecules has velocity $\mathbf{c}$ or ends up with such velocity, one has to integrate over all the possible velocities $\mathbf{c}_{1}$. The unit vector $\mathbf{e}^{\prime}$ is defined by $\mathbf{g}^{\prime} \equiv \mathbf{c}_{1}^{\prime}-\mathbf{c}^{\prime}=\left\|\mathbf{g}^{\prime}\right\| \mathbf{e}^{\prime}$, the corresponding integration over $\mathbf{e}^{\prime}$ takes into account all the possible directions that $\mathbf{c}_{1}^{\prime}$ has with respect to $\mathbf{c}^{\prime} . \Sigma(g, \chi)$ is the differential scattering cross section [48], $g \equiv\left\|\mathbf{c}_{1}-\mathbf{c}\right\|=g^{\prime} \equiv\left\|\mathbf{g}^{\prime}\right\|, \mathbf{g}=g \mathbf{e}$, and $\cos (\chi)=\mathbf{e} \cdot \mathbf{e}^{\prime}$. More details can be found in the book by Chapman and Cowling [42] and other publications [43,45-54].

Let $f=f(\mathbf{r}, \mathbf{c}, t)$ be a solution to the Boltzmann equation, then the following statements can be obtained from the Boltzmann equation, 
- For any well-behaved function (provided $f$ exists and the integrals converge) the transport equation for any function $\psi(\mathbf{r}, \mathbf{c}, t)$ is obtained by multiplying the Boltzmann equation by it and integrating over the molecular velocities,

$$
\begin{aligned}
& \int d \mathbf{c} \psi(\mathbf{r}, \mathbf{c}, t) \frac{\partial f}{\partial t}+\int d \mathbf{c} \psi(\mathbf{r}, \mathbf{c}, t) \mathbf{c} \cdot \nabla_{\mathbf{r}} f+\int d \mathbf{c} \psi(\mathbf{r}, \mathbf{c}, t) \mathbf{F} \cdot \nabla_{\mathbf{c}} f \\
= & \int d \mathbf{c} \psi(\mathbf{r}, \mathbf{c}, t) J(f, f)
\end{aligned}
$$

The following identity for the right hand side of the transport equation, Equation (33), will be useful later on and can be found in [42,47] — see Equation (3.54,5) in Reference [42] and Equation (4.1-11) in Reference [47], respectively-,

$$
\int d \mathbf{c} \psi(\mathbf{r}, \mathbf{c}, t) J(f, f)=\frac{1}{4} \int d \mathbf{c} \int d \mathbf{e}^{\prime} \int d \mathbf{c}_{1} g \Sigma(g, \chi)\left(\psi+\psi_{1}-\psi^{\prime}-\psi_{1}^{\prime}\right)\left(f^{\prime} f_{1}^{\prime}-f f_{1}\right)
$$

where the following short hand notation has been introduced for convenience,

$$
f \equiv f(\mathbf{r}, \mathbf{c}, t), \quad f_{1} \equiv f\left(\mathbf{r}, \mathbf{c}_{1}, t\right), \quad f^{\prime} \equiv f\left(\mathbf{r}, \mathbf{c}^{\prime}, t\right), \quad f_{1}^{\prime} \equiv f\left(\mathbf{r}, \mathbf{c}_{1}^{\prime}, t\right)
$$

with similar definitions for $\psi$. Equation (34) is true provided the principle of microscopic reversibility holds true.

- The most general function $\Xi(\mathbf{r}, \mathbf{c}, t)$ is a collision invariant, meaning that

$$
\int d \mathbf{c} J(f, f) \Xi(\mathbf{r}, \mathbf{c}, t)=0
$$

For a monatomic gas, a linear combination of the following fundamental collision invariants that express the mass, momentum and kinetic energy are conserved during the binary collisions:

Mass

$$
\Xi(\mathbf{r}, \mathbf{c}, t)=m
$$

Momentum

$$
\Xi(\mathbf{r}, \mathbf{c}, t)=m \mathbf{c}
$$

Energy

$$
\Xi(\mathbf{r}, \mathbf{c}, t)=\frac{m}{2}\|\mathbf{c}\|^{2}
$$

where $m$ is the mass of the molecules. For each of the previous collision invariants the transport equation given by Equation (33) leads to the conservation equations of mass, momentum, and energy, their explicit form is available in the literature $[42,45,47,48,54,56]$. Notice that for any collision invariant the right hand side of Equation (33) is zero by definition.

- For $\Psi(\mathbf{r}, \mathbf{c}, t)=\ln f(\mathbf{r}, \mathbf{c}, t)$ and defining the function $H$ by (notice that a positive distribution function is here essential),

$$
H(\mathbf{r}, t) \equiv \int d \mathbf{c} f \ln f
$$


the transport equation leads, when $\mathbf{F}=\mathbf{0}$, to

$$
\frac{\partial}{\partial t} H(\mathbf{r}, t)+\nabla \cdot\left(\mathbf{J}_{H}\right)=\sigma_{H}(\mathbf{r}, t)
$$

with,

$$
\mathbf{J}_{H}=\int d \mathbf{c c} f \ln f
$$

and

$$
\sigma_{H}(\mathbf{r}, t)=\int d \mathbf{c} J(f, f) \ln f=\frac{1}{4} \int d \mathbf{c} \int d \mathbf{e}^{\prime} \int d \mathbf{c}_{1} g \Sigma(g, \chi) \ln \left(\frac{f f_{1}}{f^{\prime} f_{1}^{\prime}}\right)\left(f^{\prime} f_{1}^{\prime}-f f_{1}\right)
$$

where the second equality in Equation (43) follows from Equation (34). The main point to notice is that the sign of $\ln \left(\frac{f f_{1}}{f^{\prime} f_{1}^{\prime}}\right)$ is always opposite to the sign of $\left(f^{\prime} f_{1}^{\prime}-f f_{1}\right)$ so that $\sigma_{H}(\mathbf{r}, t) \leq 0$ since $g \Sigma(g, \chi) \geq 0$. We are now in position to establish a connection with our previous discussion in relation with Equation (25). Defining $s(\mathbf{r}, t)=-k_{B} H(\mathbf{r}, t) / \rho(\mathbf{r}, t)$, where $k_{B}$ is the Boltzmann constant and $s$ will be identified later on, Equation (41) reads as,

$$
\frac{\partial(\rho s)}{\partial t}+\nabla \cdot\left(-k_{B} \mathbf{J}_{H}\right)=-k_{B} \sigma_{H}
$$

It turns out that in equilibrium the integral of $s$ over the volume [48] can be identified with the equilibrium entropy per unit mass of a classical perfect gas, as given by the Sackur-Tetrode formula, so that the kinetic theory of gases gives a meaning to Equation (25) when the identifications $\eta=s, \mathbf{J}_{\eta, t}=-k_{B} \mathbf{J}_{H}$, and $\sigma=-k_{B} \sigma_{H} \geq 0$, are made. It is possible to claim that since $s$ reduces to the equilibrium entropy per unit mass of a classical perfect gas, it can be considered as a generalization for situations out of equilibrium. However, being a thermodynamical concept it would be more appropriate to first show that the whole equilibrium thermodynamics admits a generalization, but this is far from being accomplished although claims contradicting this statement can be found. Furthermore, the concept of entropy has many meanings [57] and it has been pointed out [54] (p. 107) that J. von Neumann suggested Shannon to use the term entropy for a similar expression arising in statistical mechanics, arguing that nobody knew what entropy is and so in a debate he would always had the advantage. We think that the concept of entropy has a clear thermodynamic meaning within the context of LIT. Therefore, the concept of entropy production can have a well thermodynamic meaning in the same context. As we will see, the statement that the entropy production density is maximum is derived near equilibrium as happens also for LIT, but first we will make some pertinent comments.

- It is important to briefly mention Boltzmann's $H$ theorem that follows easily from what we have just seen. In fact, for an homogenous system (independent of r) Equation (41) reduces to,

$$
\frac{d H}{d t}=\sigma_{H} \leq 0
$$

which is the original $H$ theorem by Boltzmann and means that the function $H$ decreases monotonically with time. Then, since $H$ is bounded below [42] it cannot decrease indefinitely but 
tends to a limit characterized by $\frac{d H}{d t}=0$. As pointed out by Résibois-DeLenner [48] the theorem can be generalized to non-uniform systems when there is no exchange with the external world ( $\int_{A} \mathbf{J}_{H} \cdot d \mathbf{a}=0$, with $A$ the area delimiting the system) (see page 96 and also reference [47]). The condition characterizing the limit just mentioned is $\sigma_{H}=0$. It turns out that this condition holds when $\ln f$ is a collision invariant and then the local Maxwell-Boltzmann distribution function is obtained,

$$
f^{(0)}(\mathbf{r}, \mathbf{c}, t)=\frac{n(\mathbf{r}, t)}{\left(2 \pi k_{B} T(\mathbf{r}, t) / m\right)^{3 / 2}} \exp \left(-\frac{m \mathbf{c}^{2}}{2 k_{B} T(\mathbf{r}, t)}\right)
$$

where $n=\rho / m$ is the number density with $\rho$ the mass density, and $T$ is the temperature. Their expressions in terms of the distribution function are given below, see Equation (47). Some of the statements just given have not been justified with the level of rigor that mathematicians require, but detailed discussions by mathematicians of the $H$ theorem and the limit just described can be found elsewhere $[49,54]$.

In the discussion given above we started by assuming a well-behaved solution of the Boltzmann equation, this is precisely the point in which a mathematician would be interested; to give a proof that there are solutions to the Boltzmann equation, see Equation (31). The drift part of this equation, left hand side of Equation (31), does not seem to present a problem but the collision term, the right hand side of Equation (31) whose explicit form is given in Equation (32), should have a clear mathematical meaning. Here start some of the mathematical problems associated with the equation. Actually in many works a cut-off is introduced to make the collision term well-behaved since for long range potentials the total cross section diverges and this introduces mathematical problems as in the case of plasmas [55]. The problem is discussed in depth in the review by Villani [54]. According to him the theory of renormalized solutions to the Boltzmann equation introduced by DiPerna and Lions is the only proper theoretical framework in which the problem of existence can be treated in a robust way. The problem of the existence of solutions to the Boltzmann equation was considered by the famous mathematician David Hilbert (see $[46,48]$ for what we consider clear accounts of his work). The basic point that we want to stress from Hilbert's work is that he was able to show that under certain assumption on $f(\mathbf{r}, \mathbf{c}, t)$, the solution to the Boltzmann equation is determined solely in terms of the initial values of five moments; mass density $(\rho)$, hydrodynamic velocity $(\mathbf{u})$, and temperature $(T)$

$$
\rho(\mathbf{r}, 0)=m \int d \mathbf{c} f(\mathbf{r}, \mathbf{c}, 0), \quad \mathbf{u}(\mathbf{r}, 0)=\int d \mathbf{c} \mathbf{c} f(\mathbf{r}, \mathbf{c}, 0), \quad T(\mathbf{r}, 0)=\frac{m}{3 k_{B}} \int d \mathbf{c}\|\mathbf{c}\|^{2} f(\mathbf{r}, \mathbf{c}, 0)
$$

The definitions for mass density, hydrodynamic velocity, and temperature for any time follow from Equations (47) by changing the initial time $t=0$ by $t$. Hilbert's work is considered as the initial step for considering the so-called normal solutions that correspond to solutions in which the distribution is a functional of the conserved variable; $\rho(\mathbf{r}, \mathbf{c}, t), \mathbf{u}(\mathbf{r}, \mathbf{c}, t)$, and $T(\mathbf{r}, \mathbf{c}, t)$. The program was developed independently by Enskog and Chapman and the method is known as the Chapman-Enskog method for solving the Boltzmann equation [42]. The main idea is to express the solution as a power expansion in terms of the Knudsen number and to obtain the relevant equations for the different orders. To order zero in the Knudsen number the Euler equations are obtained, to first order the result is the Navier-Stokes equations, etc. Critiques to the method are available in the literature [49,54]. There are other methods 
like Grad's moments method [46], which sometimes is referred to as an uncontrolled approximation, where the main idea is to express the distribution function as a series expansion in terms of a given set of orthogonal polynomials and cut the expansion up to some point. Grad originally considered Hermite polynomials (actually tensors) and cut the expansion considering thirteen moments. Nevertheless several authors have considered more moments [58,59] or "regularizations" to Grad's thirteen moment approximation [60]. The method of the stretched fields and the Maxwellian iteration [49] have also been used for solving the Boltzmann equation. There are other methods or variants of the methods mentioned, the ones we have mentioned are the most well-known as far we know. Readers interested in knowing more about them may take a look to the bibliography [43,45-54].

For the sake of illustrating the extremum of the entropy production we will consider an example based on the Chapman-Enskog method. To determine the first order in the gradients solution with this method (first order in the Knudsen number) one arrives at a stage where such solutions written as $\Phi^{(1)}$ in $f=f^{(0)}\left(1+\Phi^{(1)}\right)$ has the form $[42,47,61]$

$$
\Phi^{(1)}=-\frac{2}{n} \mathbb{B} \mathbf{C} \mathbf{C}: \nabla \mathbf{u}-\frac{1}{n}\left(\frac{2 k_{B} T}{m}\right)^{\frac{1}{2}} \mathbf{A} \cdot \nabla \ln T
$$

where $\mathbf{C} \equiv \mathbf{c}-\mathbf{u}$ is the peculiar velocity and the tensor $\mathbb{B}$ and vector $\mathbf{A}$ satisfy the following integral equations,

$$
n I(\mathbf{A})=f^{(0)}\left(\mathscr{C}^{2}-\frac{5}{2}\right) \mathscr{C}, \quad n I(\mathbb{B})=f^{(0)} \dot{\mathscr{C}} \mathscr{C}
$$

where the vector $\mathscr{C}$ is the dimensionless peculiar velocity, $\mathscr{C} \equiv\left(\frac{m}{2 k_{B} T}\right)^{\frac{1}{2}} \mathrm{C}$, the circle denotes the corresponding traceless tensor, and the operator $I$ is given by,

$$
n^{2} I(F) \equiv \iint d \mathbf{e}^{\prime} d \mathbf{c} g \Sigma(g, \chi) f^{(0)} f_{1}^{(0)}\left(F+F_{1}-F^{\prime}-F_{1}^{\prime}\right)
$$

for any scalar function $F$ of the molecular velocity (its extension to vectors and tensor can be performed in terms of their components); the meaning of the subscripts is similar as defined in Equation (35). In the following, the bracket

$$
[F, G] \equiv \int G_{1} I(F) d \mathbf{c}_{1}
$$

will be used, when considering $[F, G]$ with $F$ and $G$ vectors (or tensors) the scalar product (or full contraction) is understood.

To proceed, one evaluates the rate of change of the local entropy of the gas $s\left(s \equiv-k_{B} \int d \mathbf{c} f \ln f\right)$ due to the collisions [62]

$$
(\partial s / \partial t)_{c o l l} \equiv \frac{1}{4} \int g b d b d \epsilon d \mathbf{c} d \mathbf{c}_{1}\left(f^{\prime} f_{1}^{\prime}-f f_{1}\right) \ln \left(f^{\prime} f_{1}^{\prime} / f f_{1}\right)
$$

using $f=f^{(0)}\left(1+\Phi^{(1)}\right)$ and keeping only linear terms in $\Phi^{(1)}$ it follows that [47]

$$
\begin{aligned}
\left(\partial s_{F K} / \partial t\right)_{c o l l} & \approx k_{B}\left(\frac{2 k_{B} T}{3 m}[\mathbf{A}, \mathbf{A}]\|\nabla \ln T\|^{2}+\frac{4}{5}[\mathbb{B}, \mathbb{B}] \mathbb{S}: \mathbb{S}\right)=\lambda\|\nabla \ln T\|^{2}+\frac{2 \mu}{T} \mathbb{S}: \mathbb{S} \\
& \equiv(\partial s / \partial t)_{c o l l, L}
\end{aligned}
$$


where $\lambda>0$ is the thermal conductivity, $\mu>0$ the shear viscosity, and $\mathbb{S}$ is the rate-of-shear tensor [47] given by,

$$
\mathbb{S}=\frac{1}{2}\left[\nabla \mathbf{u}+(\nabla \mathbf{u})^{t}\right]-\frac{1}{3} \nabla \cdot \mathbf{u} \mathbb{I}
$$

with $\mathbb{I}$ the identity matrix and the superscript $t$ denotes the transpose of the corresponding matrix.

Let a be a vector of the form $\mathbf{a}=a(C) \mathbf{C}$ with $C=\|\mathbf{C}\|$ (or $\mathbb{D}$ a tensor of the form $\mathbb{D}=D(C) \mathbf{C}$ ) that satisfies the condition $[\mathbf{a}, \mathbf{a}]=[\mathbf{a}, \mathbf{A}]$ with $\mathbf{A}=A(C) \mathbf{C}($ or $[\mathbb{D}, \mathbb{D}]=[\mathbb{D}, \mathbb{B}]$, with $\mathbb{B}=B(C) \mathbf{C C}$ ) Then, from the relation $[\mathbf{a}-\mathbf{A}, \mathbf{a}-\mathbf{A}] \geq 0$ (and a similar relation for the tensor case) it follows that,

$$
[\mathbf{a}, \mathbf{a}] \leq[\mathbf{A}, \mathbf{A}], \quad[\mathbb{D}, \mathbb{D}] \leq[\mathbb{B}, \mathbb{B}]
$$

where the equality holds when $a(C)=A(C)$ or $D(C)=B(C)$, respectively. Since $\mathbf{A}$ and $\mathbb{B}$ given in Equation (53) have the assumed forms of the statement just given, it follows that both $[\mathbf{A}, \mathbf{A}]$ and $[\mathbb{B}, \mathbb{B}]$ must have maximum values implying that $\sigma_{H}$ (see Equations (43)) (52) and (53) should also have a maximum value. In other words, we have the maximum principle (or variational principle) that the rate of change of the entropy density due to collisions is maximum. Other derivations of this maximum entropy production principle are available in the literature $[45,48]$. When the Rayleigh-Ritz method and Sonine polynomials are used, the principle provides a way to calculate the shear viscosity and thermal conductivity $[45,47,48]$. It should be pointed out that the maximum principle just given is restricted to first order in the Knudsen number and also to a linearization. In this sense, it can in no way be claimed to be more general than the Boltzmann equation from which it was obtained. There are other variational principles in the kinetic theory of gases related to the entropy production theorem discussed here, the interested reader is referred to the relevant literature $[37,63,64]$. For discussions of the entropy balance equation and extremum entropy principles using Grad's moments method, see $[65,66]$. In particular, Struchtrup and Weiss [66] proposed a minimax principle stating that the maximum over all positions of the local entropy production rate is minimal for stationary states. Indeed they consider the principle as an aid to determine the boundary conditions for the moments, and they actually considered fourteen moments in their test case. Castillo and Hoover [67,68] studied the convecting flows of a stationary, compressible, viscous and heat-conducting fluid using the Navier-Stokes equations. They found that the unstable solution with six rolls was, among the other two solutions mentioned by Castillo and Hoover, the one selected by the minimax principle. Struchtrup and Weiss replied that the boundary conditions are known for the Navier-Stokes equations and therefore there is no point for using the minimax principle in this case. While this is true we expect that any principle should give the correct answer in a simpler case, even if it is not needed, it seems that the principle does not give the correct answer for the Navier-Stokes equations. On the other hand, Weiss and Struchtrup mentioned that the minimax principle should not be used for stability analysis.

We now would like to briefly discuss the results mentioned above. First of all we would like to point out that the condition $\sigma_{H}(\mathbf{r}, t) \leq 0$, or equivalently $\left(\partial s_{F K} / \partial t\right)_{\text {coll }} \geq 0$, holds when the full solution of the Boltzmann equation is used. It may be not true when an approximation is used, in particular when the first order correction of the Chapman-Enskog method is used (first order correction in the Knudsen number) there is no guarantee that it should hold. In the maximum principle just discussed, it can be shown that when linearizing in the perturbation $\Phi$ it also holds, $\left(\partial s_{F K} / \partial t\right)_{c o l l, L} \geq 0$, but this 
could be a fortuitous accident. Closely related to this issue is another point that as far as we know has not been discussed in the literature, namely, the fact that since the perturbation is a polynomial in the components of the peculiar velocity, it is possible to conceive large values of them so that $(1+\Phi)$ is negative [46,69], implying a distribution function that can have negative values and thus $\ln f$ is not defined. Similar remarks apply to other approximations such as Grad's moment method since here, again, the approximations are polynomials in the components of the peculiar velocity. This reassures that the $H$-theorem holds when considering the exact solution of the Boltzmann equation and when using approximations one must proceed with care. For studies of entropy production, Boltzmann's $H$-theorem, the approach to equilibrium, etc., without considering approximations and from the point of view of mathematicians, see references [49,54].

We end this section discussing several points that are related with the issue at hand. We start by discussing the derivation of the Boltzmann equation using the maximum entropy (MAXENT) principle as was shown by Lewis in 1967 [70]. Actually Lewis derived not only the Boltzmann equation using the principle but he also claimed to obtain Vlasov's equation, Euler's equations, a generalization of Grad's ten moment approximation, the Gibbs distribution, Onsager's equations of irreversible thermodynamics, Liouville equation, and Hamilton's equations of classical dynamics. However, as he pointed out, he did not give an a priori justification of the principle, but an analysis that clarifies its physical content is available [71]. One question that comes to the mind is why it is possible to derive such a large number of theories from the principle and if this fact in some way compromises its predictive power. The reason for this is that Lewis' general entropy principle is based on the following assumptions [72] : (a) an entropy functional, $S[u]$, where $u$ is a state function meaning a function that describes the state of a statistical system; the $N$-particle or one particle distribution function are two examples of state functions; (b) the particle dynamics $\frac{\partial u}{\partial t}=M(u)$, with $M$ an operator which is in general nonlinear, and (c) side conditions. Thus, changing (a), (b) or (c) leads to different sets of equations which explains why it is possible to obtain different sets of equations. The problem of deriving the Boltzmann equation from the particle dynamics has also been considered by Landford [73,74], while the original proof by Landford was so short that it was not possible to justify the applications of the Boltzmann equation (for more recent discussions on this matter see reference [51]). It is interesting to notice that not all mathematicians have been interested in this problem, for example Truesdell [49] took for granted the Boltzmann equation [75]. Notice that while the Boltzmann equation is well established, its scope is limited to dilute gases, so the question arises: what to do in the case of a dense gas? This is a more delicate question and here the MAXENT method has played an important role. In fact the ideas by Lewis were extended to deal precisely with this problem. The field is known as Kinetic Variational Theories (KVT) and several kinetic equations [76-78] were derived using this methodology (see Reference [78] for more bibliography). In this field the entropy production has been explored [79] and in particular a bound to the entropy production in terms of the energy conversion rate between particles and Fisher's information integrals describing the system has been found. Using Lewis principle to obtain the kinetic description of a dense gas is not the only way to achieve the goal, and several extensions to the work by Enskog who obtained the so-called Enskog equation [48] are available. Nevertheless, a discussion of all these matters would be lengthy and somewhat distractive to our objective. 


\section{Onsager's Reciprocity Relations}

Many names of brilliant scientists come to mind in relation with the development of non-equilibrium Thermodynamics. Thus the works by Fourier, Thomson, Clausius, Einstein, and Onsager, among others, become relevant in a discussion concerning the concepts behind the entropy production. Non-equilibrium thermodynamics has its origins in the experiment and in fact, plenty of well-known irreversible effects were discovered and described before any attempt to give them a formal structure $[26,35,80]$. In a previous section the structure of usual irreversible thermodynamics was outlined, and now we will be concerned with Onsager's work in relation to the reciprocity relations [19,20,81], with emphasis on the main hypotheses driving to their demonstration. Their demonstration goes out of the thermodynamic scheme, being a result of fluctuations consideration. Here we will recall Onsager's main hypotheses in order to clarify their range of validity. First of all, let us consider an isolated macroscopic system characterized by means of a certain number of well-defined macroscopic variables $\left\{A_{i}\right\}$ which in the equilibrium state become constant. When there is a transport process occurring in the system, it manifests through time-dependent changes such that the set of variables $\left\{A_{i}(t)\right\}$ becomes time-dependent. (the selection of which and how many macroscopic variables are needed depends on the system and the quantities we are interested in). Also, we can consider the fluctuations of such variables around their equilibrium values, those fluctuations are labelled as $\alpha_{i}(t)=A_{i}-<A_{i}(t)>$ and they will be taken into account. The fluctuations are time-dependent, though in a more general treatment they can be local variables. Now, one of the hypotheses in Onsager's work is based on a direct use of the Boltzmann's fundamental relation between the entropy and the probability of the states described by the macroscopic variables $S\left(\left\{A_{i}\right\}\right)=k_{B} \ln W\left(\left\{A_{i}\right\}\right)+$ constant. Onsager considers that the entropy (or its change) is determined by the relevant variables, although the same was assumed for the entropy when written in terms of the fluctuations $\alpha_{i}$. Then,

$$
\Delta S\left(\left\{\alpha_{i}\right\}\right)=k_{B} \ln W\left(\left\{\alpha_{i}\right\}\right)
$$

It means that for a deviation of the equilibrium state involving an entropy change, the probability to observe such a deviation is proportional to $\exp \left(\Delta S / k_{B}\right)$. It should be mentioned that according to Clausius, the entropy is a state function defined for thermodynamic equilibrium states, however Equation (56) when applied to fluctuations leads the entropy concept to a non-equilibrium regime. On the other hand, the assumption made implies that this entropy obeys the same relations as it does in the equilibrium state, at least in what refers to the probability $W\left(\left\{\alpha_{i}\right\}\right)$, a hypothesis which is valid at most in the local equilibrium regime. In fact, the development in Onsager's papers begins with the structure of Linear Irreversible Thermodynamics (LIT), where the system is described by means of macroscopic variables that satisfy the balance equations. Quantities such as the heat flux, the viscous tensor, diffusion fluxes follow a set of linear phenomenological equations, in terms of the gradients in the intensive variables. In order to give the main ideas, we will restrict the discussion to variables which do not depend on position. The interested reader may found an extensive treatment in the book by Keizer [82]. Now, the definition of thermodynamic forces is given as

$$
X_{i}=\left.\left(\frac{\partial \Delta S}{\partial \alpha_{i}}\right)\right|_{\alpha_{j} \neq \alpha_{i}}=-\sum_{k} G_{i k} \alpha_{k}
$$


where $G_{i k}$ is a matrix with elements given by the second derivatives of the change in entropy with respect to the fluctuations. The corresponding fluxes are

$$
J_{i}=\frac{d \alpha_{i}}{d t}
$$

As a second step in Onsager's work he assumes that the dynamical behavior of fluctuations is described by the so-called "regression of fluctuations hypothesis", which tells us that the fluctuations follow the same behavior as the one described by the phenomenological behavior in the relevant variables.

$$
\frac{d \alpha_{i}}{d t}=J_{i}=\sum_{k} L_{i k} X_{k}=-\sum_{k j} L_{i k} G_{k j} \alpha_{j}
$$

where matrix $L_{i k}$ corresponds to the phenomenological transport coefficients. Those coefficients come from LIT and, they describe how the fluxes appear in a macroscopic system when it undergoes a transport process forced by the so-called thermodynamic forces. The regression of fluctuation hypothesis asserts that the system does not distinguish if it suffers a fluctuation or if it is forced by external means. It should also be noticed that Equation (59) implies an expansion in the change of entropy up to the second order in the fluctuations, consistent with the idea of small fluctuations around the equilibrium state. It means that this hypothesis can only be applied near the equilibrium state, where the fluctuations are small when compared with the mean value of the corresponding variable (near equilibrium are words usually employed in this subject, in this case it means that the regression of fluctuations is in the context of the local equilibrium hypothesis). Onsager remarks that those rate equations are valid for aged systems, meaning that their application is valid after a certain time $\tau_{0}$ which can be measured in terms of the Knudsen number [19,20]. The phenomenological equations containing the transport coefficients $L_{i k}$ come directly from the experiment, so their range of validity is well-defined in those terms.

All these elements allowed Onsager to derive the main result by making the most important assumption in the scheme-the "microscopic reversibility", which in the words of Onsager reads as ... if $\alpha$ and $\beta$ be two quantities which depend only on the configuration of molecules and atoms, the event $\alpha=\alpha^{\prime}$, followed $\tau$ seconds later by $\beta=\beta^{\prime}$, will occur just as often as the event $\beta=\beta^{\prime}$, followed $\tau$ seconds later by $\alpha=\alpha^{\prime}$.

$$
\overline{\alpha_{i}(t) \beta_{k}(t+\tau)}=\overline{\beta_{k}(t) \alpha_{i}(t+\tau)}
$$

The demonstration needs the averages of fluctuations at time $t$, a quantity calculated by means of Equation (56), so the relation between the entropy and probability is crucial in the development. This last hypothesis completes the scheme driving to the well-known reciprocity relations, which are fundamental in this subject. Notice that the conditions under which they were proved do not allow their extrapolation to other regimes than the one determined by the local equilibrium hypothesis and the linear relation for the constitutive equations. In a paper written by Casimir [83], he reviewed the Onsager's regression of fluctuation hypothesis and remarked that ... in principle we can imagine a pseudo-linearity holding at reasonable large amplitudes. He also generalized the Onsager's treatment to odd variables (with respect to time inversion), giving a more general proof. Regardless of this fact, it is unquestionable that the reciprocal relations represented a huge advancement, unsurpassed to date, in the development of irreversible thermodynamics. 
In connection with the results it is worth pointing that some attempts have been made to examine the validity of Onsager's reciprocity relations beyond the linear approximation. In fact, in 1982 Hurley and Garrod wrote a provocative paper [84,85] hinting at such possibility. Their arguments were basically based on the validity of a mathematical identity satisfied by an arbitrary matrix which may depend on time and of the of variables say $\left(A_{i}(\Gamma)\right)$ describing the states of the system and may be either even or odd functions of the moments. A more physical interpretation of this result was provided by a rather different approach in which the equations of motion are either Markovian or non-Markovian [86]. Later on the generalization of Onsager's reciprocity theorem (ORT) was performed including fluctuations of the regression variables around an arbitrary initial state [87]. Although in principle non-linear Markovian dynamics may be included in this formalism, concrete applications and conclusive facts that the ORT may be valid in more general cases have never been offered. The question thus remains: can the ORT be extended to a non-linear dynamics of the regression variables? This is an open question.

Going further in fluctuation theory and in connection with the relation between macroscopic and microscopic concepts, we must mention the well-known Einstein fluctuation-dissipation relation [88] which was derived in the context of the theory of Brownian motion. Later, it was generalized in a series of papers [89-91], where it was shown that there exists a close relation between the dissipation produced in an irreversible process and the correlation of fluctuations. The dissipation can be related in a direct way with the transport coefficients in the phenomenological rate equations, which are assumed to be linear. On the other hand, the correlation of fluctuations are the quantities for which Onsager has shown to obey the reciprocity relations. Hence we can say that in the linear regime, the matrix of transport coefficients and the correlation functions matrix are both symmetric, as a consequence of the microscopic reversibility introduced by Onsager. For the limitations of the Einstein relation and LIT in the context of swarms of charged particles see [30].

\section{Variational Principles}

The determination of variational principles in non-equilibrium thermodynamics has been a line of thought which does not seem to render spectacular results. The main reason for such is the presence of dissipation-when it is negligible it is possible to construct some variational principles, though they correspond to idealizations of natural phenomena. However, in the case where the dissipation is essential there exists a variational approach [92] which has been applied to numerous particular cases [93-100], which is the goal of the present section. Some emphasis must be made on the fact that the slow rate in the development of a general variational principle for non-equilibrium processes is enhanced by the presence of dissipative effects. Rayleigh [101] was the first to establish the basis for such kind of principles. In fact, he did not formulate a variational principle but instead defined the so-called dissipation function which was the basis taken by Onsager [19,102] to introduce the Principle of the Least Dissipation of Energy (LDE). The LDE takes account of the dissipation function and the entropy production as constructed by Onsager. As we said in the previous section, Onsager's treatment in non-equilibrium thermodynamics is under the frame of the local equilibrium hypothesis and the linear constitutive equations. It is then natural that his variational principle can neither be applied arbitrarily far from equilibrium nor in the case of nonlinear phenomenological equations. LDE requires the dissipation 
function written in terms of fluxes, and the entropy production is constructed from its balance equation which can also be written in terms of fluxes. Then, Onsager showed that

$$
\sigma(J)-\Phi(J, J)=\text { maximum }
$$

where $\sigma(J)=\sum_{k} J_{k} X_{k}$ corresponds to the entropy production and $\Phi(J, J)$ is the dissipation function. The variation is made up in terms of fluxes and fixed thermodynamics forces $X_{k}$. In this case the system boundaries are isolated. Such variational principle assures us that the fluxes adapt themselves to a situation in which the entropy production and the dissipation function are related by an extremal principle. The dissipation function is quadratic in the fluxes and the entropy production is consistent with linear irreversible thermodynamics, implies that it is a product of fluxes and thermodynamic forces, which leads to the conclusion that the fluxes must be linear functions of the forces. Then, the variational principle is consistent with the phenomenological constitutive equations proper to this description.

We insist that the development is valid under the assumption that the entropy production has a well-defined sense, namely, it can be written in the local equilibrium regime.

Gyarmati [11] established a generalization of the Onsager's variational principle taking into account the variation for fluxes as well as variation in thermodynamic forces, in such a way that

$$
\delta[\sigma(J, X)-\Phi(J, J)-\psi(X, X)]=0
$$

where $\psi(X, X)$ is the dissipation function expressed in terms of the thermodynamic forces. In fact, he showed that the extremum principle corresponds to a maximum and it is consistent with the linear phenomenological relations between forces and fluxes. Also, it is possible to show that a global principle is satisfied. Gyarmati takes into account that the dissipation function written in terms of fluxes as well as written in term of thermodynamic forces is quadratic and non-negative. Accordingly, the integration of the local entropy production and the local dissipation function over the entire volume of the system leads to

$$
\dot{S}_{\text {global }}=\int_{V} \sigma d V \geq 0
$$

where $\dot{S}_{\text {global }}$ contains the internal production of entropy as well as its interchange with the surroundings. Equation (63) is nothing but the Clausius inequality for the uncompensated heat, hence the development is consistent with the second law of thermostatics. Further, it is worth noticing that the demonstration was made under the frame of LIT, so it can not be extrapolated to other regimes.

In the literature the Maximum Entropy Production Principle (MEPP) proposed by Ziegler [37] has been discussed, which tells us that the entropy production written as the product of fluxes and thermodynamic forces must be a maximum. It has been applied in several examples concerning a variety of systems [103] and gives some hope about the possibility of formulating a general principle in this subject. Though this variational principle has remained somewhat hidden in the literature, it can be seen as a clue to have a new insight on some old problems. A remark on its validity is essential to give the correct perspective in the applications. Up to now, it seems to us that this principle is restricted by the same difficulties pointed out for all other principles. In fact, the proposal was made under the frame of local equilibrium thermodynamics, meaning that it is based on the entropy production written in terms of fluxes and thermodynamic forces. However, the assumption of the existence of the entropy production 
in non-equilibrium processes is taken without any question. As it was noted several times in previous sections, such an assumption assures us that it is possible to define the entropy and of course its time rate of change. If we assume that such concepts have a well defined meaning, then the proposal of having a maximal rate for the entropy production may be a useful tool to study some problems.

It should be mentioned that in the case of stationary states, a variational principle assuring that the entropy production is a minimum has been shown. In the literature there are several discussions about its extent and its general validity [38,102,104-106], in fact, mostly implying that it is valid only for stationary states under severe restrictions. Nevertheless it has been useful in several applications as well as the maximum entropy production principle [107-112].

\section{Stochastic Thermodynamics and Entropy Production}

When talking about thermodynamic behavior we usually refer to macroscopic and aged systems and, in fact, thermodynamics concepts were conceived for those cases. The corresponding microscopic treatments attempt to give support through microscopic quantities, an alternative to understand the macroscopic behavior. To achieve this goal, we usually consider systems in which the number of particles is very large $\left(N \sim 10^{23}\right)$ and times which are larger than microscopic ones, such as collision times. These requirements allow us to define a few macroscopic variables. Though they may be space and time dependent, we do not need the systems to have detailed description in terms of positions and velocities of particles. Macroscopic variables change in space and time slowly enough to make the knowledge of constituent particle coordinates unnecessary. The fluctuations of macroscopic variables have an effect on thermodynamic properties and can be measured when the system is in its equilibrium state [113-115]. Also, their spatial and temporal evolution can be followed when the system undergoes a process out of equilibrium. On the other hand, we notice that the approaches to non-equilibrium processes suffer the need of some hypothesis which so far restrict themselves to consider the problem near equilibrium. In fact, they are valid in local equilibrium but not far from it.

So, several questions arise when we consider small systems, short times and processes arbitrarily far from equilibrium $[115,121,122]$. We have two options, the first one will tell us that in such cases thermodynamic concepts have no meaning at all. Second, we can try to explore the application of thermodynamic concepts even for those cases. The interest to consider these situations arises due to the growing applications in several fields of knowledge, such as the nanotechnology. This kind of technological developments has improved the access to interesting systems such as proteins, molecular motors, colloids, etc. It is clear that the understanding of such systems not only involves the adaptation of experimental devices but also compels ourselves to revisit concepts and theoretical techniques.

Before considering the subject of fluctuations theorems as have been used to describe a thermodynamic like approach to examine their properties, a note of caution must be given. Over fifty years ago Terrel L. Hill [116-119] formulated a very precise and conventional theory to undertake the study of the thermodynamics of small systems. Such theory is based on the principles of classical thermodynamics sustained in the fundamental ideas of statistical mechanics. This is not the place to enter a detailed discussion of the theory itself nor in its various achievements, but it is worth mentioning that its various applications encompassed a wealth of systems such as colloid particles, the ideal lattice gas, the helix-coil transition and even contains a treatment of first order phase transitions. For non-equilibrium 
situations, consistent with thermodynamics, the theory is applicable for local equilibrium states. This implies that the methods for obtaining the entropy production in irreversible processes are the same as those available for large systems. Nevertheless it appears to be somewhat strange that very few efforts have been made to relate this theory to others labelled under the rather strange name of "stochastic thermodynamics", which so far pretend to describe the "thermodynamics behavior" of single "small" systems by using averaging procedures that in some way we feel ought to be equivalent to ordinary "ensemble" averages. An example of a connection of Hill's theory with the Jarzynski equality (see below) is available [120]. We leave the interested reader to consult the broad set of ideas and applications of Hill's method fully discussed in References [116,118,119].

Recently, there has been plenty of work to undertake the problem we have just outlined, and several developments have been made to describe small systems in short times when they evolve out of equilibrium. As far as we know, the genesis of the so-called "Fluctuation theorems"was the work by Evans et al. [123] and a number of papers exploring new directions emerged soon. Before going into the details, we provide a brief survey of main ideas related to such fluctuation theorems. One direction is the work followed by Gallavotti and Cohen [124,125] who enforced the "chaotic hypotheses" (also referred as strong chaocity or strong hyperbolicity) stating that many-particle systems behave like Anosov systems. The mathematical theory of dynamical systems was then used by Ruelle [126] to prove the Gallavotti-Cohen fluctuation theorem using Sinai-Ruelle-Bowen measures [127,128] where the emphasis is on non-equilibrium steady states. In order to keep the system in the steady state a thermostat is needed. Thermostats in non-equilibrium were introduced by Hoover et al. [129] and Evans et al. [130] and was an ingredient considered in the original work by Evans, Cohen, and Morris [123]. Evans and Searles [131,132] explored a different route than strong hyperbolicity and were able to prove the so-called Evans-Searles fluctuation theorem. Inspired by the experiment of Wang et al. [121], van Zon and Cohen [133,134] used Langevin dynamics to establish several work fluctuations theorems for time-dependent forces. Their work generalized the pioneering results by Kurchan [135] on the Gallavotti-Cohen fluctuation theorem for stochastic dynamics and the generalization of Kurchan's results for Markov processes by Lebowitz and Spohn [136]. Further generalizations of van Zon and Cohen's work for electromagnetic fields are available [137]. At about the same time other important lines of research were also explored, notably by Jarzynski on the one hand and Crooks on the other. Jarzynski [138] studied Hamiltonian dynamics using protocols (a specific way to drive the system from and initial to a final state) and established the so-called Jarzynski equality that relates the difference of the free energy of two equilibrium states in terms of an average that includes the work realized by the system subject to the protocol. The other is the so-called Crooks' fluctuation theorem [139] that gives the quotient of probabilities for a forward and reverse paths in terms of the difference between the work performed on the system and the free energy difference between the initial an final equilibrium states. Thus, he obtained the results assuming that the system is Markovian and microscopically reversible and from his fluctuation theorem he obtained Jarzynski's equality. Later on Crooks [140] derived a generalization of the fluctuation theorems, referring to these theorems as entropy production fluctuation theorems, and showed that the Jarzynski equality and the fluctuation theorems are connected. A comparison of the Crooks' fluctuation theorem and the one obtained by Bochkov and Kuzolev in 1977 (see [141]) has been given by Horowitz and Jarzynski [142]. There are 
other important results but their description will deviate us from the objectives of this section, so we refer the interested reader to Table 1 for more information. The concept of entropy production is an essential ingredient in some of the works just described but we think it is important to keep in mind the following remark by Dhar [143]: "An important point to note is that the definition of entropy production in small (nonthermodynamic) systems and in a non-equilibrium situation is somewhat $a d$ hoc and various definitions have been used."

Table 1. Fluctuations theorems and related research. ESFT $\equiv$ Evans-Searles fluctuation theorem, JEQ $\equiv$ Jarzynski equality, CFT $\equiv$ Crooks fluctuation theorem, BKFR $\equiv$ Bochkov-Kuzolev fluctuation relation, CWFT $\equiv$ Callen-Welton fluctuation theorem, HSEQ $\equiv$ Hatano-Sasa equality, QFT $\equiv$ Quantum fluctuation theorem, MAXENT $\equiv$ Maximum Entropy, IFT $\equiv$ Integral fluctuation theorem, GCFT $\equiv$ Gallavotti-Cohen fluctuation theorem, GSL $\equiv$ Generalization of the Second Law, MEP $\equiv$ Minimum entropy principle, ST $\equiv$ stochastic thermodynamics,

\begin{tabular}{|c|c|c|c|c|c|}
\hline $\begin{array}{l}\text { Reference } \\
\text { Experiment }\end{array}$ & $\begin{array}{c}\text { Evans-Searles } \\
\text { ESFT } \\
{[131,132]} \\
{[121]}\end{array}$ & $\begin{array}{c}\text { Jarzynski } \\
\text { JEQ } \\
{[138,144]} \\
{[152-154]} \\
\end{array}$ & $\begin{array}{c}\text { Crooks } \\
\text { CFT } \\
{[139]} \\
{[156]}\end{array}$ & $\begin{array}{c}\text { Bochkov-Kuzolev } \\
\text { BKFR } \\
{[141]}\end{array}$ & $\begin{array}{c}\text { Callen-Welton } \\
\text { CWFT } \\
{[89]}\end{array}$ \\
\hline Reference & $\begin{array}{c}\text { Hatano-Sasa } \\
\text { HSEQ } \\
\text { GSL }\end{array}$ & $\begin{array}{c}\text { Hänggi } \\
\text { QFT }\end{array}$ & $\begin{array}{c}\text { Dewar } \\
\text { MAXENT } \\
\text { MEP }\end{array}$ & $\begin{array}{l}\text { Seifert } \\
\text { IFT }\end{array}$ & $\begin{array}{c}\text { Gallavotti-Cohen } \\
\text { GCFT }\end{array}$ \\
\hline Experiment & $\begin{array}{c}{[145,146]} \\
{[155]}\end{array}$ & [147] & {$[148]$} & [149] & {$[123-126,135,136]$} \\
\hline $\begin{array}{l}\text { Reviews } \\
\text { and Books } \\
\text { Subjects }\end{array}$ & $\begin{array}{c}\text { [122] } \\
\text { ESFT, CFT }\end{array}$ & $\begin{array}{c}\text { [157] } \\
\text { JEQ, BKFR }\end{array}$ & $\begin{array}{c}{[158]} \\
\text { ST }\end{array}$ & $\begin{array}{l}159] \\
\text { GCFT }\end{array}$ & \\
\hline
\end{tabular}

According to the goal of this paper, we turn our attention to stochastic thermodynamics (a name which at least sounds somewhat curious), which is a theoretical attempt to extrapolate thermodynamic concepts to single particle trajectories $[135,150,151,158]$. Several papers report results along this line of thought, which seems to be fruitful in the applications and interpretation of experimental results mainly in nano-systems. To begin with the description made by the stochastic thermodynamics, let us first of all recall that thermodynamic quantities such as the internal energy, the entropy, etc., are state functions in equilibrium states or, slow functions of spatial coordinates and time when the system is in local equilibrium. Their fluctuations also manifest themselves through other macroscopic measurable properties. Secondly, when we see the microscopic counterpart through statistical physics, we consider ensemble averages and those averages become the quantities with a sound physical meaning. It is in this sense that stochastic thermodynamics extrapolates the usual concepts to a particle trajectory, so the trajectory quantities acquire a clear physical meaning only when averaged over the ensemble.

The simplest way to present this subject was reviewed by Seifert [158], where he considers a system formed by one particle moving in one dimension. Two kinds of forces act on the particle, one of them 
$f_{c}(x, \lambda)=-\frac{\partial V}{\partial x}$ is derived from a potential $V(x, \lambda)$ and an external force $f(x, \lambda)$, both of which may be dependent on an externally driven time-dependent protocol $\lambda(t)$. The particle is embedded in a thermal bath at temperature $T$, and the particle characteristic size is bigger than the size of the particles in the bath. Because of such conditions, it is generally assumed that the particle feels a Gaussian white noise $\zeta(t)$, with an intensity $2 D$ related to the friction $\gamma$ by means of the Einstein relation, i.e., the fluctuation-dissipation theorem is considered as a granted piece in the treatment. Hence, the bath temperature is introduced in some parts along the scheme by a relation which is valid only near equilibrium.

As mentioned by Seifert, to describe such system there are several alternatives giving equivalent descriptions. As a first approach, the particle can be described by the one dimensional overdamped Langevin equation,

$$
\begin{aligned}
\dot{x} & =\mu F(x, \lambda)+\zeta, \\
<\zeta(t) \zeta\left(t^{\prime}\right) & >=2 D \delta\left(t-t^{\prime}\right), \quad D=k_{B} T \mu
\end{aligned}
$$

where $\mu$ is the mobility and $k_{B}$ the Boltzmann constant and $F(x, \lambda)=f_{c}(x, \lambda)+f(x, \lambda)$.

Alternatively, the problem can be posed in the Fokker-Planck approach to obtain a probability density $p(x, t)$ to find the particle at position $x$ at time $t$, for a given protocol. The Fokker-Planck solution will depend on the initial distribution $p(x, 0)=p_{0}(x)$ and presumably there exists a stationary distribution $p_{s}(x, \lambda)$ for which the protocol is fixed.

$$
\frac{\partial p(x, t)}{\partial t}=-\frac{\partial}{\partial x}\left(\mu F(x, \lambda) p(x, t)-D \frac{\partial p(x, t)}{\partial x}\right)
$$

Also, the dynamical description of the particle behavior can be made in terms of a weight given to each stochastic trajectory [135]. The weight is assigned according to the Langevin dynamics. It is important to mention that the weight becomes Gaussian due to the Gaussian character assigned to the noise,

$$
P\left(x(t) \mid x_{0}\right)=\exp \left[-\int_{0}^{t}\left((\dot{x}-\mu F)^{2} / 4 D+\mu F^{\prime} / 2\right) d t^{\prime}\right]
$$

All properties which depend on the trajectory can be averaged with this weight to obtain the macroscopic variables.

This last alternative is the way chosen by stochastic thermodynamics to define the extrapolation of thermodynamic quantities. In order to give a brief description, let us consider a trajectory followed by a particle when acted by the forces, the external protocol and a given noise realization. The change in potential energy caused by a change $d x$ and a change $d \lambda$ is $d V=\frac{\partial V}{\partial x} d x+\frac{\partial V}{\partial \lambda} \dot{\lambda} d t$, and the work applied to the system $d w=\frac{\partial V}{\partial \lambda} \dot{\lambda} d t+f d x$. Now, the formal and somewhat arbitrary extrapolation of the first law of thermodynamics to the trajectory will tell us that

$$
d w=d V+d q
$$

where $d q$ plays the role of the instantaneous heat transferred along the trajectory. The direct substitution of $d V$ and the work $d w$ leads to $d q=\left(f_{c}+f\right) d x=F d x$. When it is integrated along the trajectory 
$q(x(t))=\int_{0}^{t} F\left(x, t^{\prime}\right) \dot{x} d t^{\prime}$ it gives the so-called heat transferred along the trajectory. This expression after the integration along the trajectory essentially contains the work done by the total force acting on the particle. It considers the work done by the conservative force $f_{c}$ (derived from a potential) and the work done by the external force $f$, in the presence of dissipative effects which are taken from the beginning through the overdamped Langevin equation. It is generally called as the "dissipative work"; however we must notice that it is purely mechanical work performed under dissipative effects. On the other hand, the equivalent of the change in internal energy is given by $d V$ which is also a mechanical contribution, due to the fact that the particle does not have internal degrees of freedom to account for the real internal energy (as usually understood in thermodynamics).

Once an analog of the first law is written for a trajectory, it is natural to go into what one would understand as the definition of entropy for this system. In this case it is important to take into account the environment and its change in entropy, which is assumed to be given as $\Delta \eta_{\text {env }}=\frac{q(x(t))}{T}$, where $T$ is the bath temperature. Notice that $\Delta \eta_{e n v}$ is now defined through the trajectories, since $q(x(t))$ is, hence it is also a stochastic quantity. It is therefore somewhat arbitrary and misleading to refer to it as "entropy". The system's (particle) pseudo entropy which we shall denote by $\eta$ is defined through the probability density

$$
\eta(t)=-k_{B} \ln p(x(t), t)
$$

which depends on the initial conditions contained in $p(x(t), t)$ and it is a stochastic quantity. Obviously its average over the ensemble can be written as

$$
\Xi(t)=-k_{B} \int p(x, t) \ln p(x, t) d x=<\eta(t)>
$$

and the total entropy change along a given realization of the trajectory is $\Delta \eta_{t o t}=\frac{q(x(t), t)}{T}+\Delta \eta(t)$, where $\Delta \eta(t)=-\ln p(x(t), \lambda(t))+\ln p\left(x_{0}, \lambda_{0}\right)$.

The definition of the system's "entropy" through the probability density allows the calculation of the pseudo entropy production [149]. It can be done directly by means of the Fokker-Planck equation recalling that $\dot{q}(t)=F(x, t) \dot{x}=T \dot{s}_{\text {env }}[158]$ and $\dot{\eta}_{t o t}(t)=\dot{\eta}_{e n v}+\dot{\eta}(t)$, then

$$
\dot{\eta}_{t o t}(t)=-\left.\frac{1}{p(x, t)} \frac{\partial p(x, t)}{\partial x}\right|_{x(t)}+\left.\frac{j(x, t)}{D p(x, t)}\right|_{x(t)} \dot{x}
$$

where $j(x, t)=\mu F(x, \lambda) p(x, t)-D \frac{\partial p(x, t)}{\partial x}$ is the current. The average over all trajectories which are at time $t$ at a given $x$ and the integration over all positions $x$ gives the total entropy production

$$
\dot{\Xi}_{t o t}(t)=<\dot{\eta}_{t o t}>=\int \frac{j(x, t)^{2}}{D p(x, t)} d x \geq 0
$$

which is positive and the equality holds in equilibrium. This expression deserves emphasis and some comments. First of all, Equation (68) defines a trajectory dependent "entropy" which is a stochastic quantity and it contains the information about the initial distribution. Second, the averaged "entropy" given in Equation (69) recalls us the usual Shannon's entropy definition. Third, the total entropy takes into account the heat transfer to the environment through the dissipative work as well as the entropy associated to the system itself. Fourth, the total entropy time rate, calculated according to the 
Fokker-Planck dynamics and the heat transfer with the environment is always positive or it vanishes in equilibrium. Fifth, the bath temperature plays a role in the relation between the environment entropy and the heat transfer, however it comes to the entropy production in Equation (70) when we substitute $\frac{\mu}{D}=k_{B} T$, to recognize the environment entropy production. This last comment means that such a relation is valid only as far as we can apply the fluctuation-dissipation theorem. It seems that this treatment can not be applied arbitrarily far from equilibrium. Further, all these quantities referred to as heat, entropy, etc., are debatable. To relate stochastic thermodynamics with the "fluctuation theorems", it is necessary to go further and consider again a trajectory realization. Now in the first step, consider the forward trajectory which begins in an initial position $\left(x_{0}, t=0\right)$ and ends $(x(t), t)$, for a given protocol $\lambda(t)$. The weight associated with it is given as $p\left(x(t) \mid x_{0}\right)$ as written in Equation (66), where the initial condition appears explicitly to emphasize the trajectory initial point. As a second step consider the backward trajectory $\tilde{x}(\tau)=x(t-\tau)$ which begins at $x(t)$ and ends in $x(t-\tau)$ with a protocol $\tilde{\lambda}(\tau)=\lambda(t-\tau)$. Notice that the forward and backward trajectories coincide. According to the expression of weights and the definition of forward and backward trajectories, it is possible to find the following expression,

$$
\frac{p\left(x(t) \mid x_{0}\right)}{\tilde{p}\left(\tilde{x}(t) \mid \tilde{x}_{0}\right)}=\exp \left[-\int_{0}^{t} \frac{\mu}{D} F \dot{x} d t^{\prime}\right]
$$

when the mobility $\mu$ and the noise intensity $D$ are constants. Equation (72) leads to

$$
q(x(t))=\frac{\mu}{D} \ln \frac{p\left(x(t) \mid x_{0}\right)}{\tilde{p}\left(\tilde{x}(t) \mid \tilde{x}_{0}\right)}
$$

which relates the so-called heat transfer, i.e., the dissipative work, with a quotient of probability densities in the forward and backward trajectories. Now, it becomes clear that the use of the Einstein relation $\frac{\mu}{D}=k_{B} T$ allows the introduction of the bath temperature to find the entropy production in the environment related to the dissipative work and the quotient of weights associated with the trajectories. It has been shown in the literature that this so-called entropy change satisfies the "integral fluctuation theorem"(IFT) [158],

$$
<\exp ^{-\Delta \eta_{t o t}}>=1
$$

which represents a mathematical generalization of the Clausius inequality [149,158,163-166]. In the case of a steady state under special conditions [158], it can be shown that $p\left(-\Delta \eta_{t o t}\right) / p\left(\Delta \eta_{t o t}\right)=\exp \left(-\Delta \eta_{t o t}\right)$, which tells us that the probability density decreases exponentially to observe a situation in which it appears that the second law can be violated. Taking into account that the entropy change must be an extensive quantity, the quotient of probability densities can be non-negligible for a small system and it can be measured. In fact, when we consider small systems and short scales of time, we can expect such effects, however they cannot be interpreted as violations of the second law, as usually understood [114].

Several fluctuation relations have been demonstrated experimentally, mainly for colloidal particles trapped by an optical device, some examples are given in references [121,140,152,163,164,168-171].

As it was mentioned before, the literature concerning fluctuation theorems has grown enormously, the review of such advances is out of the scope in this work and some review and recent papers are available [113,115,122,158,172-174]. Moreover, we think that it is a great mistake to borrow the 
conventional language of thermostatics to describe processes which are of an entirely different nature. It gives rise to confusion and misinterpretations.

\section{Concluding Remarks}

The main objective of this paper has been to discuss the physical meaning of the concept of entropy production in non-equilibrium processes. Introduced by De-Donder over eighty years ago it was adopted as a substitute of the more meaningful idea of non-compensated heat due to Clausius. Setting this difference aside, the question that arises is if one considers irreversible phenomena concerning how they should be dealt with. When such phenomena occur within the realm of Linear Irreversible Thermodynamics which rests in the local equilibrium assumption there are no ambiguities. The problem appears when phenomena take place "far from equilibrium", where not only this concept but also the definition of entropy itself is rather blurry. Attempts to account for this shortcoming are discussed in the paper, both macroscopically and through kinetic theory. The outcome is that in neither case we can obtain a satisfactory unique answer.

Different efforts made to cope with this situation extending Onsager's variational principle are also found in the literature but once more, all known efforts are restricted to situations in which processes occur close to equilibrium. Here we should mention the old effort set forth by Biot but no one has used it in realistic, practical phenomena.

Finally the whole body of what people now call Stochastic Thermodynamics has been critically assessed. Focused on the behavior of small systems and short times, a language has been developed extending or importing concepts from ordinary thermodynamics to situation which hardly support them. Notwithstanding the fact that the examples so far examined in the literature are of importance per se, what is very confusing is the way they are associated with ordinary thermodynamic quantities. For instance, in the case of small systems, a very powerful and solid theory based on thermodynamic and statistical mechanical principles was developed over fifty years ago. In modern literature it has been completely ignored.

Far away from equilibrium processes are still waiting for a solid theoretical framework into which they can fit. We deeply feel that such framework cannot be based in ordinary thermodynamic concepts.

\section{References and Notes}

1. Sadi-Carnot, N. Reflections on the Motive Power of Fire; Dover Publications: New York, NY, USA, 1954.

2. Thomson, W. On a Universal Tendency in Nature to the Dissipation of Mechanical Energy. Phil. Mag. 1852, 4, 304-312.

3. Newton, I. Mathematical Principles of Natural Philosophy. In Great Books of the Western World; Brittanica: Chicago, IL, USA, 1978; p. 159.

4. Clausius, R. The Mechanical Theory of Heat; Reproduction Series; Bibliobazaar: Charleston, SC, USA, 2008.

5. Cropper, W.H. Rudolf Clausius and the road to entropy. Am. J. Phys. 1986, 54, 1068-1074.

6. Planck, M. Treatise on Thermodynamics, 3rd ed.; Dover Publications: New York, NY, USA, 1945. 
7. Fourier, J.B. La Théorie Analytique du Chaleur; Editions J. Gabay: Paris, France, 1988.

8. Gibbs, J.W. The Scientific Papers of J. W. Gibbs, 1876-1878; Dover Publications: New York, NY, USA, 1961.

9. Onsager, L.; Machlup S. Fluctuations and irreversible processes. Phys. Rev. 1953, 91, 1505-1512.

10. Machlup, S.; Onsager, L. Fluctuations and Irreversible Processes II. Systems with kinetic energy. Phys. Rev. 1953, 91, 1512-1515.

11. Gyarmati, I. Non-Equilibrium Thermodynamics: Field Theory and Variational Principles; Springer-Verlag: Berlin, Germany, 1970.

12. Ziegler, H. An Introduction to Thermomechanics; North-Holland: Amsterdam, The Netherlands, 1983.

13. Biot, M.A. Variational Principle in Heat Transfer; Oxford Mathematical Monographs Series; Oxford Press: Clarendon, TX, USA, 1970.

14. Glansdorff, P.; Prigogine, I. Thermodynamic Theory of Structure, Stability and Fluctuations (Part II); Wiley-Interscience: New York, NY, USA, 1971.

15. de Donder, T. L'Affinité. (Part 1); Gauthier-Vilars: Paris, France, 1928.

16. de Donder, T. L’Affinité. (Part 2); Gauthier-Vilars: Paris, France, 1931.

17. Garfinkle, M. Natural Path in Chemical Thermodynamics. J. Chem. Phys. 1989, 93, 2158-2164.

18. Garfinkle, M. The thermodynamic Natural Path in chemical reaction kinetics. Discrete Dynam. Nature Soc. 2000, 4, 145-164.

19. Onsager, L. Reciprocal relations in irreversible processes. I. Phys. Rev. 1931, 37, 405-426.

20. Onsager, L. Reciprocal relations in irreversible processes. II. Phys. Rev. 1931, 38, 2265-2279.

21. Eckart, C. The Thermodynamics of Irreversible Processes I. The Simple Fluid. Phys. Rev. 1940, 58, 267-269.

22. Eckart, C. The Thermodynamics of Irreversible Processes II. Fluid Mixtures. Phys. Rev. 1940, $58,269-275$.

23. Meixner, J. Zur Thermodynamik der irreversiblen Prozesse. Z. Physik Chem. 1943, 53, 235-263.

24. Prigogine, I. Introduction to Themodynamics of Irreversible Processes; Charles C. Thomas: Springfield, IL, USA, 1955.

25. de Groot, S.R. Thermodynamics of Irreversible Processes; North-Holland: Amsterdam, The Netherlands, 1952.

26. de Groot, S.R.; Mazur, P. Non-Equilibrium Thermodynamics; North-Holland: Amsterdam, The Netherlands, 1962.

27. Hutter, K. The Foundations of Thermodynamics, its Basic Postulates and Implications. A Review of Modern Thermodynamics. Acta Mech. 1977, 27, 1-54.

28. Hutter, K.; Wang, Y. Phenomenological Thermodynamics and Entropy Principles. In Entropy; Princeton Series in Applied Mathematics; Greven, A., Keller, G., Warnecke, G., Eds.; Princeton University Press: Princeton, NJ, USA, 2003; pp. 55-75.

29. Müller, I. Entropy in Nonequilibrium. In Entropy; Princeton Series in Applied Mathematics; Greven, A., Keller, G., Warnecke, G., Eds.; Princeton University Press: Princeton, NJ, USA, 2003; pp. 79-104. 
30. García-Colín, L.S.; Uribe, F.J. Extended Irreversible Thermodynamics Beyond the Linear Regime: A Critical Overview. J. Non-Equilib. Thermodyn. 1991, 16, 89-128.

31. García-Colín, L.S.; del Río, J.L. Onsager Reciprocity Relations: The Connection Between Microphysics and Macrophysics. Rev. Mex. Fis. 1993, 39, 669-684.

32. Meixner, J. The Entropy problem in thermodynamics and processes. Reol. Acta 1973, 12, 465-467.

33. García-Colín, L.S.; del Río, J.L. Concept of entropy for nonequilibrium states of closed many-body systems. Phys. Rev. A 1991, 41, 6657-6663.

34. García-Colín, L.S.; del Río, J. L. Increase-in-entropy law. Phys. Rev. E 1993, 48, 819-828.

35. Tolman, R.C.; Fine, P.C. On the Irreversible Production of Entropy. Rev. Mod. Phys. 1948, 20, 51-77.

36. Županović, P.; Juertié, D.; Botrić, S. Kirchhoff's loop law and the maximum entropy production principle. Phys. Rev. E 2004, 70, 056108.

37. Martyushev, L.M.; Seleznev, V.D. Maximum entropy production principle in physics, chemistry and biology. Phys. Rep. 2006, 426, 1-45.

38. Jaynes, E.T. The Minimum Entropy Production Principle. Ann. Rev. Phys. Chem. 1980, 31, 579-601.

39. Jaynes, E.T. Where do we stand on Maximum Entropy. In The Maximum Entropy Principle; Levine, R., Tribus, M., Eds.; MIT Press: Cambridge, MA, USA, 1978; p. 1.

40. Hoover, Wm.G. Time Reversibility, Computer Simulation, and Chaos; World Scientific: Singapore, 1999.

41. Our source is the book by Chapman and Cowling [42] and in particular the historical summary that appears in the third edition. More detailed information can be found in the preface by S. G. Brush to the english translation of the book by Boltzmann originally entitled "Vorlesungen über Gastheorie" [43] and the works by Brush referred therein like reference [44].

42. Chapman, S.; Cowling, T.G. The Mathematical Theory of Non-Uniform Gases; Cambridge University Press: Cambridge, UK, 1970.

43. Boltzmann, L. Lectures on Gas Theory; Brush, S.G., trans.; Dover: New York, NY, USA, 1995.

44. Brush, S.G. Statistical Physics and the Atomic Theory of Matter from Boyle and Newton to Landau and Onsager; Princeton University Press; Princeton, NJ, USA, 1983.

45. Hirschfelder, J.O.; Curtiss, C.F.; Bird, R.B. Molecular Theory of Gases and Liquids; John Wiley and Sons: New York, NY, USA, 1954.

46. Grad, H. Principles of the kinetic theory of gases. In Handbuch der Physik; Flügge, D., Ed.; Springer-Verlag: Berlin, Germany, 1958, pp. 205-294.

47. Ferziger, J.H.; Kaper, H.P. Mathematical Theory of Transport Processes in Gases; North-Holland: Amsterdam, The Netehrlands, 1972; p. 133.

48. Résibois, P.; de Leener, M. Classical Kinetic Theory of Fluids; John Wiley \& Sons: New York, NY, USA, 1977.

49. Truesdell, C.; Muncaster, R.G. Fundamentals of Maxwell's Kinetic Theory of a Simple Monatomic Gas; Academic Press: New York, NY, USA, 1980. 
50. McCourt, F.R.W.; Bennakker, J.J.; Köhler, W.E.; Kuščer, I. Nonequilibrium Phenomena in Polyaomic Gases; Oxford Science Publications: Oxford, UK, 1990.

51. Cercignani, C.; Illner, R.; Pulvirenti, M. The Mathematical Theory of Dilute Gases; Springer-Verlag: New York, NY, USA, 1994.

52. Eu, B.C. Nonequilibrium Statistical Mechanics. Ensemble Method; Kluwer: Dordrecht, The Netherlands, 1998.

53. Cercignani, C. The Boltzmann equation and fluid dynamics. In Handbook of Mathematical Fluid Dynamics; Friedlander, S., Serre, D., Eds.; Elsevier Science: Amsterdam, The Netherlands, 2002; Volume 1.

54. Villani, C. A review of mathematical topics in collisional kinetic theory. In Handbook of Mathematical Fluid Dynamics; Friedlander, S., Serre, D., Eds.; Elsevier Science, Amsterdam, The Netherlands, 2002; Volume 1.

55. García-Colín, L.S.; Dagdug, L. Kinetic Theory of Dilute Inert Plasmas; Springer: Berlin, Germany, 2009.

56. García-Colín, L.S.; Velasco, R.M.; Uribe, F.J. Beyond the Navier-Stokes equations: Burnett hydrodynamics. Phys. Rep. 2008, 465, 149-189.

57. Grad, H. The many faces of entropy. Comm. Pure Appl. Math. 1961, 14, 323-354.

58. Müller, I.; Ruggeri, T. Extended Thermodynamics; Springer: New York, NY, USA, 1993.

59. Weiss, W. Continuous shock structure in extended thermodynamics. Phys. Rev. E 1995, 52, R5760-R5763.

60. Struchtrup, H. Macroscopic Transport Equations for Rarefied Gas Flows; Springer-Verlag: Berlin, Germany, 2005.

61. The vector $\mathbf{A}$ and tensor $\mathbb{B}$ used by Ferziger and Kaper [47] are different from the corresponding quantities given in the book by Chapman and Cowling [42].

62. The form of collision operator is now expressed in terms of the impact parameter, see [42,47] for more details.

63. Snider, R.F. Variational Methods for Soving the Boltzmann Equation. J. Chem Phys. 1964, 41, 591-595.

64. The "principle of maximum entropy production" given here is attributed [37] to Enskog in his doctoral dissertation of 1917, see [37] for more references.

65. Velasco, R.M.; García-Colín, L.S. The Kinetic Foundations of Extended Irreversible Thermodynamics Revisited. J. Stat. Phys. 1992, 69, 217-229.

66. Struchtrup, H.; Weiss, W. Maximum of the Local Entropy Production Becomes Minimal in Stationary Processes. Phys. Rev. E 1998, 80, 5048-5051.

67. Castillo, V.M.; Hoover, W.G. Comment on "Maximum of the Local Entropy Production Becomes Minimal in Stationary Processes". Phys. Rev. Lett. 1998, 81, 5700.

68. Struchtrup, H.; Weiss, W. Struchtrup and Weiss Reply, Phys. Rev. Lett. 1998, 81, 5701. 
69. This can be seen as follows: take for simplicity the case in which the hydrodynamic velocity is zero and that the temperature depends only on the coordinate $x$. Then to first order in the Chapman-Enskog method the distribution function is given by $f=f^{(0)}(1+\Phi)$ with

$$
\Phi=-\frac{1}{n}\left(\frac{2 k_{B} T}{m}\right)^{\frac{1}{2}} \frac{A(|\mathscr{C}|)}{T} \mathscr{C}_{x} \frac{d T}{d x}
$$

with $\mathscr{C}$ the reduced peculiar velocity and $A(|\mathscr{C}|)$ is an scalar function. If we take its first Sonine expansion [42] (Chapter 7) it has the form

$$
A(|\mathscr{C}|)=-\frac{2 m \lambda}{5 k_{B}^{2} T}\left(\frac{5}{2}-|\mathscr{C}|^{2}\right)
$$

Choosing $\mathscr{C}_{x}$ large enough (either positive or negative) for given values of the thermal conductivity $(\lambda), n, T$ and its gradient, we can make $1+\Phi$ negative so that $\ln f=\ln \left(f^{(0)}(1+\Phi)\right)$ is not defined.

70. Lewis, R.M. A Unifying Principle in Statistical Mechanics. J. Math. Phys. 1997, 8, 1448-1459.

71. del Río-Correa, J.L.; García-Colín, L.S.; Micenmacher, V. Lewis principle revisited. Phys. A 1995, 219, 361-374.

72. We consider here Lewis' general principle which is used to derive the Boltzmann equation. The special principle follows from the general one [70].

73. Landford, O.E. On a Derivation of the Boltzmann Equation; Springer Lecture Notes in Physcis 38; Springer-Verlag: Berlin, Germany, 1975; pp. 1-111.

74. Landford, O.E., III. On a Derivation of the Boltzmann Equation. In Nonequilibrium Phenomena I: The Boltzmann Equation; Lebowitz, J.L.; Montroll, E.M., Eds.; North-Holland: Amsterdam, The Netherlands, 1983.

75. The terminology used by Truesdell is somewhat different by the one used by physicists. This actually makes difficult to read Truesdells works for physicists. In particular he does not use the term Boltzmann equation but he refers to as the Maxwell-Boltzmann equation.

76. Karkheck, J. Maximization of entropy, kinetic-equations, and irreversible thermodynamics. Phys. Rev. A 1982, 25, 3302-3327.

77. Stell, G. Kinetic mean field-theories-results of energy constrain in maximazing entropy. J. Chem. Phys. 1983, 79, 3166-3167.

78. Romero-Salazar, L.; Mayorga, M.; Velasco, R.M. Maximum entropy formalism for a dense gas. Physica A 1997, 327, 150-168.

79. Mayorga, M.; Romero-Salazar, L.; Velasco, R.M. Entropy production bound in a dense gas. Phys. A 1997, 246, 145-156.

80. Müller, I. A History of Thermodynamics. The Doctrine of Energy and Entropy; Springer-Verlag: Berlin, Germany, 2007; pp. 233-272.

81. Lax, M. Fluctuations from the Nonequilibrium Steady State. Rev. Mod. Phys. 1960, 32, 25-64.

82. Keizer, J. Statistical Thermodynamics of Nonequilibrium Processes; Springer-Verla: New York, NY, USA, 1987.

83. Casimir, H.B.G. On Onsager's Principle of Microscopic Reversibility. Rev. Mod. Phys. 1945, 17, 343-350. 
84. Hurley, J.; Garrod, C. Generalization of the Onsager Reciprocity Theorem. Phys. Rev. Lett. 1982, $48,1575-1577$.

85. Garrod, C.; Hurley, J. Symmetry-Relations for the Conductivity Tensor. Phys. Rev. A 1983, 27, 1487-1490.

86. García-Colín, L.S.; del Rio, J.L. Further Generalization of the Onsager Reciprocity Theorem. Phys. Rev. A 1984, 30, 3314-3316.

87. García-Colín, L.S.; Rodriguez, R.F. Microscopic Derivation of the Onsager Reciprocity Relations. Phys. Rev. A 1987, 36, 4945-4949.

88. Einstein, A. Investigations on the Theory of the Brownian Movement; Dover: New York, NY, USA, 1956.

89. Callen, H.B.; Welton, T.A. Irreversibility and Generalized Noise. Phys. Rev. 1951, 83, 34-40.

90. Callen, H.B; Greene, R.F. On a Theorem of Ireversible Thermodynamics. Phys. Rev. 1952, 86, 702-710.

91. Greene, R.F.; Callen, H.B. On a Theorem of Irreversible Thermodynamics. II. Phys. Rev. 1952, 88, 1387-1391.

92. Berdichevsky, V.L. Variational Principles of Continuum Mechanics I. Fundamentals; Springer: Berlin, Germany, 2009; Chapter 12.

93. Sieniutycz, S.; Farkas, H. Variational and Extremum Principles in Macroscopic Systems; Elsevier: Amsterdam, The Netherlands, 2005.

94. Virgo, N. From Maximum Entropy to Maximum Entropy Production: A New Approach. Entropy 2010, 12, 107-126.

95. Dyke, J.; Kleidon, A. The Maximum Entropy Production Principle: Its Theoretical Foundations and Applications to the Earth System. Entropy 2010, 12, 613-630.

96. Županovic, P.; Kuic, D.; Juretic, D.; Dobovisek, A. On the Problem of Formulating Principles in Nonequilibrium Thermodynamics. Entropy 2010, 12, 926-931.

97. Županovic, P.; Kuic, D.; Losic, Z.B.; Petrov, D.; Juretic, D. Brumen, M. The Maximum Entropy Production Principle and Linear Irreversible Processes. Entropy 2010, 12, 996-1005.

98. Favretti, M. The Maximum Entropy Rate Description of a Thermodynamic System in a Stationary Non-Equilibrium State. Entropy 2009, 11, 675-687.

99. Dewar, R.C. Maximum Entropy Production as an Inference Algorithm that Translates Physical Assumptions into Macroscopic Predictions: Don't Shoot the Messenger. Entropy 2009, 11, 931-944.

100. Paltridge, G.W. A Story and a Recommendation about the Principle of Maximum Entropy Production. Entropy 2009, 11, 945-948.

101. Strutt, R.W. Some General Theorems relating to Vibrations. Proc. Math. Soc. London 1873, 4, 357-368.

102. Hillert, M.; Ågren, J. Extremum principles for irreversible processes. Acta Mater. 2006, 54, 2063-2066.

103. Kleidon, A.; Lorenz, R.D. Non-Equilibrium Thermodynamics and the Production of Entropy; Springer: Berlin, Germany, 2005. 
104. Prigogine, I. Introduction to Thermodynamics of Irreversible Processes; Interscience: New York, NY, USA, 1967.

105. Glansdorff, P.; Prigogine, I. On a General Evolution Criterion in Macroscopic Physics. Physica 1934, 30, 351-374.

106. Klein, M.J.; Meijer, P.H. Principle of Minimum Entropy Production. Phys. Rev. 1954, 96, $250-255$.

107. Christen, T. Modeling Electric Discharges with Entropy Production Rate Principle. Entropy 2009, 11, 1042-1054.

108. Salamon, P; Nitzan, A.; Andresen, B.; Berry, R.S. Minimum entropy production and the optimization of heat engines. Phys. Rev. A 1980, 21, 2115-2129.

109. Benilov, M.S.; Naidis, G.V. What is the mathematical meaning of Steenbeck's principle of minimum power in gas discharge physics? J. Phys. D Appl. Phys. 2010, 43, 175204.

110. Di Vita, A. Maximum or minimum entropy production? How to select a necessary criterion of stability for a dissipative fluid or plasma. Phys. Rev. E 2010, 81, 041137.

111. Garbet, X.; Dubuit, N.; Asp, E.; Sarazin, Y.; Bourdelle, C.; Ghendrih, P.; Hoang, G.T. Turbulet fluxes and entropy production rate. Phys. Plasm. 2005, 12, 082511.

112. Vázquez, F.; Olivares-Robles, M.A.; Cuevas, S. Viscoelastic Effects on the Entropy Production in Oscillatory Flow between Parallel Plates with Convective Cooling. Entropy 2009, 11, 4-16.

113. Harris, R.J.; Schütz, G.M. Fluctuation theorems for stochastic dynamics. J. Stat. Mech. 2007, $\mathrm{P} 07020$.

114. Grandy, W.T. Entropy and the Time Evolution of Macroscopic Systems; Oxford University Press: Oxford, UK, 2008.

115. Marconi, U.M.B.; Puglisi, A.; Rondoni, L.; Vulpiani, A. Fluctuation-dissipation: Response theory in statistical physics. Phys. Rep. 2008, 461, 111-195.

116. Hill, T.L. Thermodynamics of Small Systems, Part I ; Benjamin: New York, NY, USA, 1963.

117. Hill, T.L. Thermodynamics of Small Systems, Part II; Benjamin: New York, NY, USA, 1964.

118. Hill, T.L. Thermodynamics for Chemist and Biologist; Addison Wesley: Reading, MA, USA, 1968; Chapter 6.

119. Hill, T.L. On 1st-order Phase Transitions in Canonical and Grand Ensembles. J. Chem. Phys. 1955, 23, 812-816.

120. Carrete, J.; Varela, L.M.; Gallego, L.J. Nonequilibrium nanothermodynamics. Phys. Rev. E 2008, 77, 022102.

121. Wang, G.M.; Sevick, E.M.; Mittag, E.; Searles, D.J.; Evans, D.J. Experimental demonstration of violations of the second law of thermodynamics for small systems and short time scales. Phys. Rev. Lett. 2002, 89, 050601.

122. Sevick, E.M.; Prabhakar, R.; Williams, S.R.; Searles, D.J. Fluctuation theorems. Annu. Rev. Phys. Chem. 2008, 59, 603-633.

123. Evans, D.J.; Cohen, E.G.D.; Morris, G.P. Probability of 2nd law violations in shearing steady-state. Phys. Rev. Lett. 1993, 71, 2401-2404.

124. Gallavotti, G.; Cohen, E.G.D. Dynamical ensembles in nonequilibrium statistical mechanics. Phys. Rev. Lett. 1995, 74, 2694-2697. 
125. Gallavotti, G.; Cohen, E.G.D. Dynamical ensembles in stationary states. J. Stat. Phys. 1995, 80, 931-970.

126. Ruelle, D. Smooth Dynamics and New Theoretical Ideas in Nonequilibrium Statistical Mechanics. J. Stat. Phys. 199, 95, 393-468.

127. Eckman, J.-P.; Ruelle, D. Ergodic theory of chaos and strange attractors. Rev. Mod. Phys. 1985, 57, 617-656.

128. Young, L.-S. What Are SRB Measures, and Which Dynamical Systems Have Them? J. Stat. Phys. 2002, 108, 733-754.

129. Hoover, W,G.; Ladd, A.J.C.; Moran, B. High-Strain-Rate Plastic Flow Studied via Nonequilibrium Molecular Dynamics. Phys. Rev. Lett. 1982, 48, 1818-1820.

130. Evans, D.J.; Hoover; W.G.; Failor, B.H.; Moran, B.; Ladd, A.J.C. Nonequilibrium molecular dynamics via Gauss's principle of least constrain. Phys. Rev. A 1983, 28, 1016-1021.

131. Evans, D.J.; Searles, D.J. Equilibrium microstates which generate second law violating steady states. Phys. Rev. E 1994, 50, 1645-1648.

132. Evans, D.J.; Searles, D.J. The fluctuation theorem. Adv. Phys. 2002, 51, 1529-1585.

133. van Zon, R.; Cohen, E.G.D. Stationary and transient work-fluctuation theorems for a dragged Brownian particle. Phys. Rev. E 2003, 67, 046102.

134. van Zon, R.; Cohen, E.G.D. Extended heat-fluctuation theorems for a system with deterministic and stochastic forces. Phys. Rev. E 2004, 69, 056121.

135. Kurchan, J. Fluctuation theorem for stochastic dynamics. J. Phys. A Math. Gen. 1998, 31, 3719-3729.

136. Lebowitz, J.L.; Spohn, H. A Gallavotti-Cohen-Type Symmetry in the Large Deviation Functional for Stochastic Dynamics. J. Stat. Phys. 1999, 95, 333-365.

137. Jiménez-Aquino, J.I.; Uribe, F.J.; Velasco, R.M. Work-fluctuation theorems for a particle in an electromagnetic field. J. Phys. A Math. Theor. 2010, 43, 255001.

138. Jarzynski, C. Nonequilibrium equality for free energy differences. Phys. Rev. Lett. 1997, 78, 2690-2693.

139. Crooks, G.E. Nonequilibrium measurements of free energy differences for microscopically reversible Markovian systems. J. Stat. Phys. 1998, 90, 1481-1487.

140. Crooks, G.E. Entropy production fluctuation theorem and the nonequilibrium work relation for free energy differences. Phys. Rev. E 1999, 60, 2721-2726.

141. Bochkov, G.N.; Kuzovlev, Y.E. Non-linear fluctuation-dissipation relations and stochastic models in non-equlibrium thermodynamics.1. Generalized fluctuation-dissipation theorem. Physica 1981, 106, 443-479.

142. Horowitz, J.; Jarzynski, C. Comparisons of work fluctuation relations. J. Stat. Phys. Theor. Exp. 2009, P11002.

143. Dhar, A. Work distribution functions in polymer stretching experiments. Phys. Rev. E 2005, 71, 036126.

144. Hummer, G.; Szabo, A. Free Energy Reconstruction from Nonequilibrium Single-Molecule Pulling Experiments. Proc. Nat. Am. Sci. 2001, 98, 3658-3661. 
145. Hatano, T. Jarzynski equality for the transitions between nonequilibrium steady states. Phys. Rev. E 1999, 60, R5017-R5020.

146. Hatano, T.; Sasa, S.I. Steady-state thermodynamics of Langevin systems. Phys. Rev. Lett. 2001, 86, 3463-3466.

147. Campisi, M.; Talkner, P.; Hänggi, P. Fluctuation Theorem for Arbitrary Open Quantum Systems. Phys. Rev. Lett. 2009, 102, 210401.

148. Dewar, R. Information theory explanation of the fluctuation theorem, maximum entropy production and self-organized criticality in non-equilibrium stationary states. J. Phys. A Math. Theor. 2005, 38, L371-L381.

149. Seifert, U. Entropy Production along a Stochastic Trajectory and an Integral Fluctuation Theorem. Phys. Rev. Lett. 2005, 95, 040602.

150. Taniguchi, T.; Cohen, E.G.D. Onsager-Machlup Theory for Nonequilibrium Steady States and Fluctuation Theorems. J. Stat. Phys. 2006, 126, 1-41.

151. Chernyak, V.Y.; Chertov, M.; Jarzynski, C. Path-integral analysis of fluctuation theorems for general Langevin processes. J. Stat. Mech. 2006, P08001.

152. Liphardt, J.; Dumont, S.; Smith, S.B.; Tinoco, I.; Bustamente, C. Equilibrium information from nonequilibrium measurements in an experimental test of Jarzynski's equality. Science 2002, 296, 1832-1835.

153. Douarche, F.; Ciliberto, S.; Patrosyan, A.; Rabbiosi, I. An experimental test of the Jarzynski equality in a mechanical experiment. Europhys. Lett. 2005, 70, 593-599.

154. Huber, G.; Schmidt-Kaler, F.; Deffner, S.; Lutz, E. Employing trapped cold ions to verify the quantum Jarzynski equality. Phys. Rev. Lett. 2008, 101, 070403.

155. Trepagnier, E.H.; Jarzynski, C.; Ritort, F.; Crooks, G.E.; Bustamante, C.J.; Liphardt, J. Experimental test of Hatano and Sasa's nonequilibrium steady-state equality. Proc. Nat. Am. Sci. 2004, 101, 15038-15041.

156. Collin, D.; Ritort, F.; Jarzynski, C.; Smith, S.B.; Tinoco, I., Jr.; Bustamante, C. Verification of the Crooks fluctuation theorem and recovery of RNA folding free energies. Nature 2005, 437, 231-234.

157. Jarzynski, C. Comparison of far-from-equilibrium work relations. C. R. Physique 2007, 8, 495-506.

158. Seifert, U. Stochastic thermodynamics: Principles and perspectives. Eur. Phys. J. B 2008, 64, 423-431.

159. Jiang, D-Q.; Qian, M.; Qian, M-P. Mathematical Theory of Nonequilibrium Steady States; Springer-Verlag: Berlin, Germany, 2004.

160. Evans, D.J.; Morris, G.P. Statistical Mechanics of Nonequilibrium Liquids; Academic Press: San Diego, CA, USA, 1990.

161. Cohen, E.G.D.; Gallavotti, G. Note on Two Theorems in Nonequilibrium Statistical Mechanics. J. Stat. Phys. 1999, 96, 1343-1349.

162. Abhisheck, D. Work distribution functions in polymer stretching experiments. Phys. Rev. E 2005, $71,036126$. 
163. Ge, H.; Jiang, D-Q. The transient fluctuation theorem of a sample entropy production for general stochastic processes. J. Phys. A Math. Theor. 2007, 40, F713-F723.

164. Lahiri, S.; Jayannavar, A.M. Total entropy production fluctuation theorems in a nonequilibrium time-periodic steady state. Eur.Phys. J. B 2009, 69, 87-92.

165. Saha, A.; Lahiri, S.; Jayannavar, A.M. Entropy production theorems and some consequences. Phys. Rev. E 2009, 80, 011117.

166. Imparato, A.; Peliti, L. Fluctuation relation for a driven Brownian particle. Phys. Rev. E 2006, 74, 026106.

167. Baiesi, M.; Jacobs, T.; Maes, C.; Skantzos, N.S. Fluctuation symmetries for work and heat. Phys. Rev. E 2006, 74, 021111.

168. Blickle, V.; Speck, T.; Helden, L.; Seifert, U. Bechinger, C. Thermodynamics of a colloidal particle in a time-dependent nonharmonic potential. Phys. Rev. Lett. 2006 96, 070603.

169. Carberry, D.M.; Reid, J.C.; Wang, G.M., Sevick, E.M.; Searles, D.J.; Evans, D.J. Fluctuations and Irreversibility: An experimental demonstration of a second-law-like theorem using a colloidal particle held in an optical trap. Phys. Rev. Lett. 2004, 92, 140601.

170. Joubaud, S.; Garnier, N.B.; Ciliberto, S. Fluctuation theorems for harmonic oscillators. J. Stat. Mech. Theory Experiment 2007, P09018.

171. Chetrite, R.; Falkovich, G.; Gawedzki, K. Fluctuation relations in simple examples of non-equilibrium steady states. J. Stat. Mech. 2008, P08005.

172. Evans, D.J.; Rondoni, L. Comments on the entropy of nonequilibrium steady states. J. Stat. Phys. 2002, 109, 895-920.

173. Jimenez-Aquino, J.I. Entropy production theorem for a charged particle in an electromagnetic field. Phys. Rev. E 2010, 82, 051118.

174. Hoover, W.G.; Hoover, C.G. Nonequilibrium Fluctuations in a Gaussian Galton Board (or Periodic Lorentz Gas) Using Long Period Orbits. ArXiv [cond-mat.stat-mech] 2010, 0143477.

(c) 2011 by the authors; licensee MDPI, Basel, Switzerland. This article is an open access article distributed under the terms and conditions of the Creative Commons Attribution license (http://creativecommons.org/licenses/by/3.0/.) 